\title{
Rhodium-Catalyzed Reductive Aldol Reactions Using Aldehydes as the Stoichiometric Reductants.
}

\author{
Michael C. Willis* and Robert L. Woodward \\ ${ }^{a}$ Department of Chemistry, University of Bath, Bath, BA2 7AY, UK.
}

\section{Supporting Information}

General Information: IR spectra were recorded on a Perkin-Elmer 1600 FT IR spectrophotometer, using $\mathrm{NaCl}$ discs or as solids on a Perkin-Elmer Spectrum One FT-IR spectrometer opperating in Attenuated Total Reflectance mode. ${ }^{1} \mathrm{H}$ NMR spectra were obtained on a Bruker Avance 300 spectrometer operating at $300 \mathrm{MHz}$, unless otherwise noted, with tetramethylsilane as an internal standard. $J$ values are given in $\mathrm{Hz} .{ }^{13} \mathrm{C}$ NMR spectra were obtained on a Bruker Avance 300 spectrometer operating at $75 \mathrm{MHz}$, unless otherwise noted. All dry solvents were freshly distilled under nitrogen prior to use. Dichloroethane was distilled over calcium hydride, tetrahydrofuran was distilled over sodium wire. Petroleum ether refers to that fraction obtained between $40-60^{\circ} \mathrm{C}$. All glassware was dried in an oven and allowed to cool under nitrogen prior to use. All commercial reagents were used as obtained, except for 3-(methylthio)propionaldehyde which was distilled before use.

Mass spectrometry measurements were performed at the EPSRC National Mass Spectrometry Service Centre, University of Wales Swansea; values are quoted as $\mathrm{m} / \mathrm{z}$ with relative intensity in parentheses.

Thin layer chromatographic analyses were performed on plates coated with Kieselgel $60 \mathrm{~F}_{254}$. Visualisation was achieved with a $254 \mathrm{~nm}$ ultraviolet lamp, followed by staining with vanillin or potassium permanganate. Column chromatographic separation was carried out using silica gel (35-70 mesh).

\section{Preparation of $\beta$-Sulfide-Substituted Aldehydes}

Aldehydes were prepared according to the method of Weber, ${ }^{1}$ with the following minor modifications: A mixture of the enal $(1 \mathrm{~mol})$ and thiol $(1.25 \mathrm{~mol})$ were stirred at room temperature before addition of sodium hydride $(0.5 \mathrm{~mol} \%)$ in four portions over 15 
minutes. Care, extremely exothermic reaction ensues. The resultant reaction mixture was left to stir at room temperature for a further 3-10 hours before removal of the volatile components and distillation at reduced pressure to afford the $\beta$-(alkylthio)aldehydes as colourless oils.

General procedure for the reductive aldol reactions (Table 1, Entry 1)

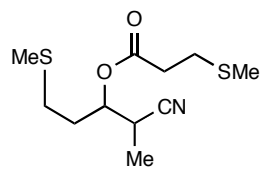

Hydrogen gas was bubbled through a stirred solution of $[\mathrm{Rh}(\mathrm{nbd}) \mathrm{dppe}] \mathrm{ClO}_{4}(10 \mathrm{mg}$, $0.014 \mathrm{mmol})^{2}$ dissolved in DCE $(10 \mathrm{~mL})$ for 2 minutes, during this time a color change from dark orange to yellow was realised, the solution was then purged with argon for a further 0.5 minutes. To this mixture was next added 3-(methylthio)propionaldehyde (14 $\mu \mathrm{L}, 15 \mathrm{mg}, 0.14 \mathrm{mmol})$ and acrylonitrile $(46 \mu \mathrm{L}, 37 \mathrm{mg}, 0.7 \mathrm{mmol})$. The reaction mixture was then heated to $70^{\circ} \mathrm{C}$ for 16 hours. The crude reaction mixture was concentrated in vacuo and purified by column chromatography (3:1 hexane: EtOAc) to give the product as a colorless oil $(16 \mathrm{mg}, 88 \%)$ as a mixture of diastereomers (2.3:1); $\delta_{\mathrm{H}}\left(300 \mathrm{MHz}, \mathrm{CDCl}_{3}\right.$ ) (Major diastereomer) $5.08(1 \mathrm{H}, \mathrm{ddd}, J$ 8.0, 4.2, $4.2 \mathrm{OCH}), 2.90$ $(1 \mathrm{H}, \mathrm{m}, \mathrm{CHCN}), 2.76-2.70\left(2 \mathrm{H}, \mathrm{m}, \mathrm{CH}_{2} \mathrm{CH}_{2} \mathrm{CO}_{2}\right), 2.66-2.61\left(2 \mathrm{H}, \mathrm{m}, \mathrm{CH}_{2} \mathrm{CH}_{2} \mathrm{CO}_{2}\right), 2.07$ ( $\left.3 \mathrm{H}, \mathrm{s}, \mathrm{SCH}_{3}\right), 1.98-1.83\left(2 \mathrm{H}, \mathrm{m}, \mathrm{CH}_{2} \mathrm{CHO}\right), 1.27$ (3H, d, J 7.4, $\mathrm{CHCH}_{3}$ ); (Minor diastereomer) $4.96(1 \mathrm{H}, \mathrm{ddd}, J 9.0,5.0,3.0, \mathrm{OCH}), 2.99(1 \mathrm{H}, \mathrm{qd}, \mathrm{CHCN}), 2.76-2.70(2 \mathrm{H}$, m, $\left.\mathrm{CH}_{2} \mathrm{CH}_{2} \mathrm{CO}_{2}\right), 2.66-2.61\left(2 \mathrm{H}, \mathrm{m}, \mathrm{CH}_{2} \mathrm{CH}_{2} \mathrm{CO}_{2}\right), 2.04(3 \mathrm{H}, \mathrm{s}, \mathrm{SCH}), 1.98-1.83(2 \mathrm{H}, \mathrm{m}$, $\left.\mathrm{CH}_{2} \mathrm{CHO}\right), 1.27\left(3 \mathrm{H}, \mathrm{d}, \mathrm{J} 7.4, \mathrm{CHCH}_{3}\right) ; \delta_{\mathrm{C}}\left(75 \mathrm{MHz}, \mathrm{CDCl}_{3}\right)$ 170.3, 118.8, 70.9, 33.2, 30.6, 29.3, 28.8, 28.0, 14.5, 13.5; $v_{\max }$ (film) / $\mathrm{cm}^{-1} 2244,1732 ; \mathrm{m} / \mathrm{z}\left(\mathrm{CI}+, \mathrm{NH}_{3}\right) 279(100$ $\%, \mathrm{M}+\mathrm{NH}_{4}$ ); found $279.1196\left[\mathrm{M}+\mathrm{NH}_{4}\right]^{+} \mathrm{C}_{11} \mathrm{H}_{23} \mathrm{~N}_{2} \mathrm{O}_{2} \mathrm{~S}_{2}$ requires 279.1195 . 
Table 1, Entry 2

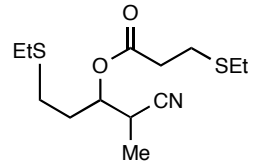

The typical procedure was followed to afford the product as a colorless oil $(16 \mathrm{mg}, 79 \%)$ as a mixture of diastereomers $(3.3: 1) ; \delta_{\mathrm{H}}\left(300 \mathrm{MHz}, \mathrm{CDCl}_{3}\right)(\mathrm{Major}$ diastereomer) $5.07(1 \mathrm{H}, \mathrm{m} \mathrm{OCH}), 3.04-2.85(1 \mathrm{H}, \mathrm{m}, \mathrm{CHCN}), 2.79-2.71\left(2 \mathrm{H}, \mathrm{m}, \mathrm{CH}_{2} \mathrm{~S}\right)$, 2.66-2.56 (10H, m, $\left.\mathrm{CH}_{2} \mathrm{~S} / \mathrm{CH}_{2} \mathrm{C}(\mathrm{O})\right), 2.09-1.81\left(2 \mathrm{H}, \mathrm{m}, \mathrm{CH}_{2} \mathrm{CHOC}(\mathrm{O})\right), 1.27(3 \mathrm{H}, \mathrm{d}, J$ 7.2 $\left.\mathrm{CH}_{3} \mathrm{CHCN}\right), 1.20\left(3 \mathrm{H}, \mathrm{t}, J 7.5, \mathrm{SCH}_{2} \mathrm{CH}_{3}\right), 1.19\left(3 \mathrm{H}, \mathrm{t}, J 7.5, \mathrm{SCH}_{2} \mathrm{CH}_{3}\right)$; (Minor diastereomer) $4.96(1 \mathrm{H}, \mathrm{m} \mathrm{OCH}), 3.04-2.85(1 \mathrm{H}, \mathrm{m}, \mathrm{CHCN}), 2.79-2.71\left(2 \mathrm{H}, \mathrm{m}, \mathrm{CH}_{2} \mathrm{~S}\right)$, 2.66-2.56 (10H, m, $\left.\mathrm{CH}_{2} \mathrm{~S}, \mathrm{CH}_{2} \mathrm{C}(\mathrm{O})\right), 2.09-1.81$ (2H, m, $\left.\mathrm{CH}_{2} \mathrm{CHOC}(\mathrm{O})\right), 1.27$ (3H, d, J 7.2 $\left.\mathrm{CH}_{3} \mathrm{CHCN}\right), 1.20\left(3 \mathrm{H}, \mathrm{t}, J\right.$ 7.5, $\left.\mathrm{SCH}_{2} \mathrm{CH}_{3}\right), 1.19\left(3 \mathrm{H}, \mathrm{t}, J 7.5, \mathrm{SCH}_{2} \mathrm{CH}_{3}\right) ; \delta_{\mathrm{C}}(75 \mathrm{MHz}$, $\left.\mathrm{CDCl}_{3}\right)$ 171.72, 171.66, 120.2, 119.9, 72.7, 72.4, 35.1, 32.6, 30.8, 30.7, 27.6, 26.9, 26.40, 26.35, 26.35, 26.32, 15.08, 15.05, 15.0, 14.9, 14.7; $v_{\max }\left(\right.$ film) / $\mathrm{cm}^{-1} 2928,1740,1452$; $\mathrm{m} / \mathrm{z}(\mathrm{CI}+) 307\left(100 \%, \mathrm{M}+\mathrm{NH}_{4}\right), 290,245$; found $[\mathrm{M}+\mathrm{H}]^{+} 290.1243 \mathrm{C}_{13} \mathrm{H}_{24} \mathrm{NO}_{2} \mathrm{~S}_{2}$ requires 290.1243 .

\section{Table 1, Entry 3}

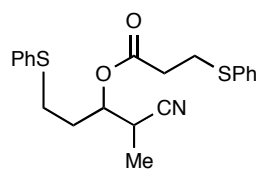

The typical procedure was followed to afford the product as a colorless oil $(21 \mathrm{mg}, 78 \%)$ as a mixture of diastereomers $(2.5: 1) ; \delta_{\mathrm{H}}\left(300 \mathrm{MHz}, \mathrm{CDCl}_{3}\right)\left(\mathrm{Major}^{2}\right.$ diastereomer) 7.40-7.16 (10H, m, $\mathrm{Ph} \times 2), 5.17(1 \mathrm{H}, \mathrm{ddd}, J$ 7.5, 7.5, 7.2, OCH), 3.22-3.13 $\left(2 \mathrm{H}, \mathrm{m}, \mathrm{CH}_{2} \mathrm{SPh}\right), 3.13-2.81\left(3 \mathrm{H}, \mathrm{m}, \mathrm{CH}_{2} \mathrm{SPh} / \mathrm{CHCN}\right), 2.70\left(2 \mathrm{H}, \mathrm{t}, J\right.$ J $\left..2, \mathrm{CH}_{2} \mathrm{CO}_{2}\right), 2.18-$ $1.88\left(2 \mathrm{H}, \mathrm{m}, \mathrm{CH}_{2} \mathrm{CHOC}(\mathrm{O})\right), 1.30\left(3 \mathrm{H}, \mathrm{d}, \mathrm{J} 7.2 \mathrm{CH}_{3} \mathrm{CHCN}\right)$; (Minor diastereomer) 7.40$7.16(10 \mathrm{H}, \mathrm{m}, \mathrm{Ph} \times 2), 5.06(1 \mathrm{H}, \mathrm{ddd}, J 7.5,7.5,7.2, \mathrm{OCH}), 3.22-3.13\left(2 \mathrm{H}, \mathrm{m}, \mathrm{CH}_{2} \mathrm{SPh}\right)$, 3.13-2.81 (3H, m, $\left.\mathrm{CH}_{2} \mathrm{SPh} / \mathrm{CHCN}\right), 2.67\left(2 \mathrm{H}, \mathrm{t}, J\right.$ 7.2, $\left.\mathrm{CH}_{2} \mathrm{CO}_{2}\right), 2.18-1.88(2 \mathrm{H}, \mathrm{m}$, $\left.\mathrm{CH}_{2} \mathrm{CHOC}(\mathrm{O})\right), 1.26\left(3 \mathrm{H}, \mathrm{d}, \mathrm{J} 7.2 \mathrm{CH}_{3} \mathrm{CHCN}\right) ; \delta_{\mathrm{C}}\left(75 \mathrm{MHz}, \mathrm{CDCl}_{3}\right)$ 171.1, 171.06, 135.1, 134.7, 130.41, 130.37, 129.93, 129.91, 129.14, 129.10, 126.9, 126.6, 119.7, 119.4, 72.1, $71.8,34.2,34.19,32.0,30.4,30.2,29.8,29.7,29.2,14.5,14.2 ; v_{\max }\left(\right.$ film) $/ \mathrm{cm}^{-1} 2922$, 
1740, 1439; m/z (EI+) 385 (19\%, M+), 275, 203; found $[\mathrm{M}+\mathrm{H}]^{+} 386.1247 \mathrm{C}_{21} \mathrm{H}_{24} \mathrm{NO}_{2} \mathrm{~S}_{2}$ requires 386.1243; (Found C, 65.4; H, 6.0, N 3.6. $\mathrm{C}_{21} \mathrm{H}_{23} \mathrm{NO}_{2} \mathrm{~S}_{2}$ requires C, 65.4; H, 6.0; $\mathrm{N}, 3.6)$.

\section{Table 1, Entry 4}

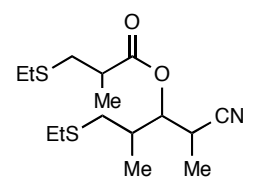

The typical procedure was followed to afford the product as a colorless oil $(19 \mathrm{mg}, 88 \%)$ as a mixture of diastereomers (A:B:C, 6.6:3.6:1.0); $\delta_{\mathrm{H}}\left(300 \mathrm{MHz}, \mathrm{CDCl}_{3}\right)$ (Diastereomer A) $5.00(1 \mathrm{H}, \mathrm{m}, \mathrm{OCH}), 3.06-2.90(1 \mathrm{H}, \mathrm{m}, \mathrm{CHCN}), 2.58-2.10(10 \mathrm{H}, \mathrm{m}$, $\left.\mathrm{SCH}_{2} \mathrm{CH}_{3} / \mathrm{CH}_{2} \mathrm{CHC}(\mathrm{O})\right), 1.32-1.15\left(12 \mathrm{H}, \mathrm{m}, \mathrm{SCH}_{2} \mathrm{CH}_{3} / \mathrm{CH}_{2} \mathrm{CHCH}_{3}\right), 1.07$ (3H, d, $J$ 6.8, $\left.\mathrm{CH}_{3} \mathrm{CHCN}\right)$; (Diastereomer B) 4.84 (1H, m, OCH), 3.06-2.90 (1H, m, CHCN), 2.58$2.10\left(10 \mathrm{H}, \mathrm{m}, \mathrm{SCH}_{2} \mathrm{CH}_{3} / \mathrm{CH}_{2} \mathrm{CHC}(\mathrm{O})\right), 1.32-1.15\left(12 \mathrm{H}, \mathrm{m}, \mathrm{SCH}_{2} \mathrm{CH}_{3} / \mathrm{CH}_{2} \mathrm{CHCH}_{3}\right)$, 1.02 (3H, d, J 6.8, $\left.\mathrm{CH}_{3} \mathrm{CHCN}\right)$; (Diastereomer C) $5.19(1 \mathrm{H}, \mathrm{m}, \mathrm{OCH}), 3.06-2.90(1 \mathrm{H}, \mathrm{m}$, $\mathrm{CHCN}), 2.58-2.10\left(10 \mathrm{H}, \mathrm{m}, \mathrm{SCH}_{2} \mathrm{CH}_{3} / \mathrm{CH}_{2} \mathrm{CHC}(\mathrm{O})\right), 1.32-1.15\left(12 \mathrm{H}, \mathrm{m}, \mathrm{SCH}_{2} \mathrm{CH}_{3} /\right.$ $\left.\mathrm{CH}_{2} \mathrm{CHCH}_{3}\right), 1.09\left(3 \mathrm{H}, \mathrm{d}, J\right.$ 6.8, $\left.\mathrm{CH}_{3} \mathrm{CHCN}\right) ; \delta_{\mathrm{C}}\left(75 \mathrm{MHz}, \mathrm{CDCl}_{3}\right)$ 173.5, 173.3, 118.8, $118.3,74.3,74.1,73.5,39.41,39.35,35.1,34.9,34.8,34.7,34.5,34.2,34.0,33.7,33.61$, $33.57,32.6,27.9,27.5,27.3,25.6,25.4,25.3,25.2,16.3,16.2,16.1,16.0,14.5,14.3$, 14.2, 13.6, 13.5; $v_{\max }\left(\right.$ film) / $\mathrm{cm}^{-1} 2971,2929,2361,1741,1458 ; \mathrm{m} / \mathrm{z}(\mathrm{CI}+) 335$ (100\%, $\left.\mathrm{M}+\mathrm{NH}_{4}\right), 318,273$; found $[\mathrm{M}+\mathrm{H}]^{+} 318.1558 \mathrm{C}_{15} \mathrm{H}_{28} \mathrm{NO}_{2} \mathrm{~S}_{2}$ requires 318.1556 .

\section{Table 1, Entry 5}

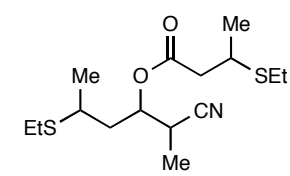

The typical procedure was followed to afford the product as a colorless oil (16 mg, $73 \%$ ) as a mixture of diastereomers (A:B:C, 7.5:5:1); $\delta_{\mathrm{H}}\left(300 \mathrm{MHz}, \mathrm{CDCl}_{3}\right)$ (Diastereomer A) 5.14-5.06 (1H, m, OCH), 3.25-3.09 (1H, m, CHCN), 3.09-2.83 (1H, m, $\mathrm{CH}_{3} \mathrm{CHSEt}$ ), 2.83-2.65 (1H, m, CHSEt), 2.59-2.41 (6H, m, $\mathrm{SCH}_{2} \mathrm{CH}_{3} / \mathrm{CH}_{2} \mathrm{CO}_{2}$ ), 2.17$1.60\left(2 \mathrm{H}, \mathrm{m}, \mathrm{CH}_{2} \mathrm{CHOC}(\mathrm{O})\right), 1.34-1.12\left(12 \mathrm{H}, \mathrm{m}, \mathrm{CHCH}_{3} / \mathrm{SCH}_{2} \mathrm{CH}_{3}\right)$; (Diastereomer B) 
5.29-5.21 (1H, m, OCH), 3.25-3.09 (1H, m, CHCN), 3.09-2.83 (1H, m, $\left.\mathrm{CH}_{3} \mathrm{CHSEt}\right)$, 2.83-2.65 (1H, m, CHSEt), 2.59-2.41 (6H, m, SCH $\left.\mathrm{CH}_{3} / \mathrm{CH}_{2} \mathrm{CO}_{2}\right), 2.17-1.60$ (2H, m, $\left.\mathrm{CH}_{2} \mathrm{CHOC}(\mathrm{O})\right), 1.34-1.12\left(12 \mathrm{H}, \mathrm{m}, \mathrm{CHCH}_{3} / \mathrm{SCH}_{2} \mathrm{CH}_{3}\right.$ ); (Diastereomer C) 4.99-4.91 $(1 \mathrm{H}, \mathrm{m}, \mathrm{OCH}), 3.25-3.09$ (1H, m, CHCN), 3.09-2.83 (1H, m, $\left.\mathrm{CH}_{3} \mathrm{CHSEt}\right), 2.83-2.65$ (1H, m, CHSEt), 2.59-2.41 (6H, m, $\mathrm{SCH}_{2} \mathrm{CH}_{3} / \mathrm{CH}_{2} \mathrm{CO}_{2}$ ), 2.17-1.60 (2H, m, $\mathrm{CH}_{2} \mathrm{CHOC}(\mathrm{O})$ ), 1.34-1.12 (12H, m, $\left.\mathrm{CHCH}_{3} / \mathrm{SCH}_{2} \mathrm{CH}_{3}\right) ; \delta_{\mathrm{C}}\left(75 \mathrm{MHz}, \mathrm{CDCl}_{3}\right)$ 171.2, 171.1, 120.09, 120.06, 72.2, 71.6, 71.3, 71.2, 42.5, 40.5, 40.4, 39.7, 39.6, 37.6, 36.9, 36.8, 36.2, 35.8, $35.7,31.4,31.3,31.0,30.7,30.6,30.1$, 24.9, 24.82, 24.78, 24.7, 24.25, 24.16, 23.23, 23.17, 22.0, 21.8, 21.5, 20.8; $v_{\max }\left(\right.$ film) $/ \mathrm{cm}^{-1} 2968,2728,2244,1740 ; \mathrm{m} / \mathrm{z}$ (EI+) 317 $(13 \%, \mathrm{M}+), 288,255$; found $317.1476[\mathrm{M}+\mathrm{H}]^{+} \mathrm{C}_{15} \mathrm{H}_{28} \mathrm{NO}_{2} \mathrm{~S}_{2}$ requires 317.1477.

\section{Table 1, Entry 6}

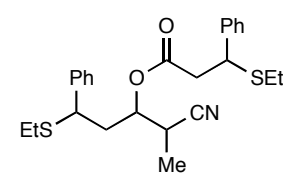

The typical procedure was followed to afford the product as a colorless oil $(22 \mathrm{mg}, 71 \%)$ as a mixture of diastereomers $(1.1: 1) ; \delta_{\mathrm{H}}\left(300 \mathrm{MHz}, \mathrm{CDCl}_{3}\right)(\mathrm{Major}$ Diastereomer) 7.35-7.08 (9H, m, $\mathrm{Ph} \times 2), 7.00-6.88(1 \mathrm{H}, \mathrm{m}, \mathrm{Ph}), 5.12-5.02(1 \mathrm{H}, \mathrm{m}$, $\mathrm{OCH}), 4.28-4.18(1 \mathrm{H}, \mathrm{m} \mathrm{SCHPh}), 3.77-3.61(1 \mathrm{H}, \mathrm{m}, \mathrm{CHCN}), 2.85-2.52\left(2 \mathrm{H}, \mathrm{m} \mathrm{C}_{\mathrm{H} 2} \mathrm{CO}_{2}\right)$, 2.36-2.10 (4H, m, $\left.\mathrm{SCH}_{2} \mathrm{CH}_{3}\right), 2.10-1.92\left(2 \mathrm{H}, \mathrm{m}, \mathrm{CH}_{2} \mathrm{CHO}\right), 1.23-0.95\left(6 \mathrm{H}, \mathrm{m} \mathrm{CH}_{3} \mathrm{CH}_{2} \mathrm{~S}\right)$, 0.90-0.77 (3H, d, J 7.2, $\left.\mathrm{CH}_{3} \mathrm{CHCN}\right)$; (Minor Diastereomer) 7.35-7.08 (10H, m, $\mathrm{Ph} \times 2$ ), 4.70-4.60 (1H, m, OCH), 4.18-4.10 (1H, m SCHPh), 3.63-3.50 (1H, m, CHCN), 2.85$2.52\left(2 \mathrm{H}, \mathrm{m} \mathrm{C}_{\mathrm{H} 2} \mathrm{CO}_{2}\right), 2.36-2.10\left(4 \mathrm{H}, \mathrm{m}, \mathrm{SCH}_{2} \mathrm{CH}_{3}\right), 2.10-1.92\left(2 \mathrm{H}, \mathrm{m}, \mathrm{CH}_{2} \mathrm{CHO}\right), 1.23-$ $0.95\left(6 \mathrm{H}, \mathrm{m} \mathrm{CH}_{3} \mathrm{CH}_{2} \mathrm{~S}\right), 0.90-0.77\left(3 \mathrm{H}, \mathrm{d}, J 7.2, \mathrm{CH}_{3} \mathrm{CHCN}\right) ; \delta_{\mathrm{C}}\left(75 \mathrm{MHz}, \mathrm{CDCl}_{3}\right)$ 170.2, 170.1, 141.5, 141.4, 129.11, 129.05, 128.3, 128.22, 128.17, 128.1, 119.8, 71.2, 45.5, 41.8, 30.7, 25.61, 25.55, 25.46, 14.73, 14.70; $v_{\max }$ (film) / $\mathrm{cm}^{-1} 2963,2921,2244,1742,1453$; $\mathrm{m} / \mathrm{z}(\mathrm{CI}+) 459\left(25 \%, \mathrm{M}+\mathrm{NH}_{4}\right), 442,399,249$; found $442.1873[\mathrm{M}+\mathrm{H}]{ }^{+} \mathrm{C}_{25} \mathrm{H}_{32} \mathrm{O}_{2} \mathrm{~S}_{2} \mathrm{~N}$ requires 442.1869 . 
Table 1, Entry 7

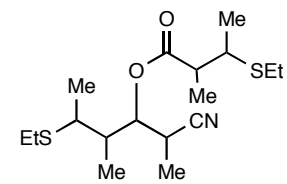

The typical procedure was followed to afford the product as a colorless oil $(22 \mathrm{mg}, 93 \%)$ as a mixture of diastereomers $(1.4: 1) ; \delta_{\mathrm{H}}\left(300 \mathrm{MHz}, \mathrm{CDCl}_{3}\right)(\mathrm{Major}$ diastereomer) 5.15-5.02 (1H, m, OCH), 3.20-2.75 (2H, m, CHCN / $\left.\mathrm{CH}_{3} \mathrm{CHC}(\mathrm{O})\right)$, 2.742.42 (3H, m, $\left.\mathrm{CH}_{3} \mathrm{CHS} / \mathrm{CH}_{3} \mathrm{CHOC}(\mathrm{O})\right), 1.57-1.51$ (3H, m, $\left.\mathrm{CH}_{3} \mathrm{CHC}(\mathrm{O})\right), 1.35-1.20$ (15H, m, $\mathrm{CH}_{3} \mathrm{CH}_{2} \mathrm{~S} / \mathrm{CH}_{3}$ ), 0.87-0.74 (3H, m, $\mathrm{CH}_{3} \mathrm{CN}$ ); (Minor Diastereomer) 5.69-5.57 $(1 \mathrm{H}, \mathrm{m}, \mathrm{OCH}), 3.20-2.75$ (2H, m, $\left.\mathrm{CHCN} / \mathrm{CH}_{3} \mathrm{CHC}(\mathrm{O})\right), 2.74-2.42$ (3H, m, $\mathrm{CH}_{3} \mathrm{CHS} /$ $\left.\mathrm{CH}_{3} \mathrm{CHOC}(\mathrm{O})\right), 1.57-1.51\left(3 \mathrm{H}, \mathrm{m}, \mathrm{CH}_{3} \mathrm{CHC}(\mathrm{O})\right), 1.35-1.20\left(15 \mathrm{H}, \mathrm{m}, \mathrm{CH}_{3} \mathrm{CH}_{2} \mathrm{~S} / \mathrm{CH}_{3}\right)$, 0.87-0.74 (3H, m, $\left.\mathrm{CH}_{3} \mathrm{CN}\right) ; \delta_{\mathrm{C}}\left(75 \mathrm{MHz}, \mathrm{CDCl}_{3}\right)$ 175.1, 174.93, 174.91, 120.4, 120.3, 75.95, 75.93, 75.1, 41.02, 40.95, 36.7, 36.2, 36.1, 35.3, 35.2, 29.5, 29.1, 27.2, 27.04, 26.99, 26.91, 26.8, 18.0, 17.8, 16.2, 15.9,15.8, 15.22, 15.18, 15.1, 14.9; $v_{\max }\left(\right.$ film) / cm $\mathrm{cm}^{-1}$ 2973, 2921, 2244, 1739, 1452; m/z (CI+) 363 (49\%, M+NH $\left.{ }_{4}\right), 301,284,201$; found $346.1870[\mathrm{M}+\mathrm{H}]^{+} \mathrm{C}_{17} \mathrm{H}_{32} \mathrm{NO}_{2} \mathrm{~S}_{2}$ requires 346.1869.

\section{Table 1, Entry 8}

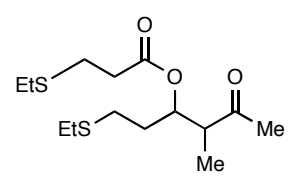

The typical procedure was followed to afford the product as a colorless oil $(16 \mathrm{mg}, 73 \%)$ as a mixture of diastereomers $(1.4: 1) ; \delta_{\mathrm{H}}\left(300 \mathrm{MHz}, \mathrm{CDCl}_{3}\right)(\mathrm{Major}$ diastereomer) 5.26-5.17 (1H, m, OCH), 2.80-2.65 (3H, m, CHCN / $\left.\mathrm{CH}_{2} \mathrm{CO}_{2}\right), 2.58-2.38$ $\left(6 \mathrm{H}, \mathrm{m}, \mathrm{SCH}_{2}\right), 2.13,\left(3 \mathrm{H}, \mathrm{s}, \mathrm{CH}_{3} \mathrm{C}(\mathrm{O})\right), 1.85-1.70\left(2 \mathrm{H}, \mathrm{m}, \mathrm{CH}_{2} \mathrm{CHOC}(\mathrm{O})\right), 1.25-1.13$ (6H, m, $\left.\mathrm{CH}_{3} \mathrm{CH}_{2} \mathrm{~S}\right), 1.05$ (3H, d, J 7.2, $\mathrm{CH}_{3} \mathrm{CHCN}$ ); (Minor Diastereomer) 5.34-5.25 $(1 \mathrm{H}, \mathrm{m}, \mathrm{OCH}) 2.14(1 \mathrm{H}, \mathrm{m}, \mathrm{CHCN}), 2.80-2.65\left(2 \mathrm{H}, \mathrm{m}, \mathrm{CH}_{2} \mathrm{CO}_{2}\right), 2.58-2.38(6 \mathrm{H}, \mathrm{m}$, $\left.\mathrm{SCH}_{2}\right), 2.14,\left(3 \mathrm{H}, \mathrm{s}, \mathrm{CH}_{3} \mathrm{C}(\mathrm{O})\right), 1.85-1.70\left(2 \mathrm{H}, \mathrm{m}, \mathrm{CH}_{2} \mathrm{CHOC}(\mathrm{O})\right), 1.25-1.13(6 \mathrm{H}, \mathrm{m}$, $\left.\mathrm{CH}_{3} \mathrm{CH}_{2} \mathrm{~S}\right), 1.04\left(3 \mathrm{H}, \mathrm{d}, J\right.$ 7.2, $\left.\mathrm{CH}_{3} \mathrm{CHCN}\right) ; \delta_{\mathrm{C}}\left(75 \mathrm{MHz}, \mathrm{CDCl}_{3}\right)$ 209.51, 209.47, 171.9, 171.8, 74.0, 73.6, 50.63, 50.60, 35.3, 35.2, 34.6, 32.5, 31.1, 30.8, 29.7, 28.1, 27.7, 27.0, 26.9, 26.6, 26.4, 36.3, 25.9, 23.1, 15.09, 15.07, 12.0, 11.7; $v_{\max }$ (film) / $\mathrm{cm}^{-1} 2971,2928$, 
2103, 1734, 1714, 1644; ; m/z (CI+) $324\left(100 \%, \mathrm{M}+\mathrm{NH}_{4}\right), 307,262,190$; found $307.1398[\mathrm{M}+\mathrm{H}]^{+} \mathrm{C}_{14} \mathrm{H}_{27} \mathrm{O}_{3} \mathrm{~S}_{2}$ requires 307.1396.

\section{Table 1, Entry 9}

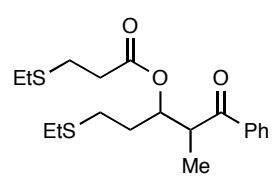

The typical procedure was followed to afford the product as a colorless oil $(19 \mathrm{mg}, 74 \%)$ as a mixture of diastereomers $(3.3: 1) ; \delta_{\mathrm{H}}\left(300 \mathrm{MHz}, \mathrm{CDCl}_{3}\right)$ (Major diastereomer) 8.01-7.95 (2H, m, Ph), 7.56-7.47 (1H, m, Ph), 7.46-7.37 (2H, m, Ph), 5.37$5.24(1 \mathrm{H}, \mathrm{m}, \mathrm{OCH}), 3.87\left(1 \mathrm{H}\right.$, quint, $\left.J 6.8, \mathrm{CHCH}_{3}\right), 2.71-2.61\left(2 \mathrm{H}, \mathrm{m}, \mathrm{SCH}_{2} \mathrm{CH}_{3}\right), 2.52-$ $2.31\left(8 \mathrm{H}, \mathrm{m}, \mathrm{SCH}_{2} / \mathrm{CH}_{2} \mathrm{CO}_{2}\right), 1.88-1.74\left(2 \mathrm{H}, \mathrm{m}, \mathrm{CH}_{2} \mathrm{CHOC}(\mathrm{O})\right), 1.21-1.08(9 \mathrm{H}, \mathrm{m}$, $\left.\mathrm{SCH}_{2} \mathrm{CH}_{3}\right) / \mathrm{CHCH}_{3}$ ); (Minor Diastereomer) 7.87-7.82 (2H, m, Ph), 7.56-7.47 (1H, m, $\mathrm{Ph}), 7.46-7.37(2 \mathrm{H}, \mathrm{m}, \mathrm{Ph}), 5.37-5.24(1 \mathrm{H}, \mathrm{m}, \mathrm{OCH}), 3.75\left(1 \mathrm{H}\right.$, quint, $\left.J 6.8, \mathrm{CHCH}_{3}\right)$, 2.71-2.61 (2H, m, $\left.\mathrm{SCH}_{2} \mathrm{CH}_{3}\right), 2.52-2.31\left(8 \mathrm{H}, \mathrm{m}, \mathrm{SCH}_{2} / \mathrm{CH}_{2} \mathrm{CO}_{2}\right), 1.88-1.74(2 \mathrm{H}, \mathrm{m}$, $\left.\left.\mathrm{CH}_{2} \mathrm{CHOC}(\mathrm{O})\right), 1.21-1.08\left(9 \mathrm{H}, \mathrm{m}, \mathrm{SCH}_{2} \mathrm{CH}_{3}\right) / \mathrm{CHCH}_{3}\right) ; \delta_{\mathrm{C}}\left(75 \mathrm{MHz}, \mathrm{CDCl}_{3}\right)$ 201.9, 201.3, 171.9, 171.8, 137.0, 136.7, 129.2, 129.0, 128.7, 128.5, 75.0, 74.8, 44.3, 44.2, 35.3, $35.2,32.6,32.5,30.5,28.1,27.8,26.91,26.86,26.4,26.29,26.25,26.15,15.1,14.1$, $12.2 ; v_{\max }($ film $) / \mathrm{cm}^{-1} 2969,2928,2103,1734,1682,1448 ; \mathrm{m} / \mathrm{z}$ (CI+) $386(52 \%$, $\left.\mathrm{M}+\mathrm{NH}_{4}\right), 369,252,235$; found $369.1550[\mathrm{M}+\mathrm{H}]^{+} \mathrm{C}_{19} \mathrm{H}_{29} \mathrm{O}_{3} \mathrm{~S}_{2}$ requires 369.1553 .

\section{Table 1, Entry 10}

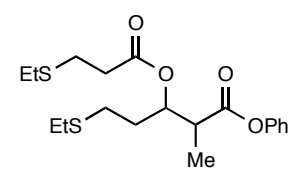

The typical procedure was followed to afford the product as a colorless oil $(18 \mathrm{mg}, 67 \%)$ as a mixture of diastereomers $(3.3: 1) ; \delta_{\mathrm{H}}\left(300 \mathrm{MHz}, \mathrm{CDCl}_{3}\right)$ (Major diastereomer) 7.35-7.25 (2H, m, Ph), 7.21-7.12 (1H, m, Ph), 7.05-6.96 (2H, m, Ph), 5.44$5.29(1 \mathrm{H}, \mathrm{m}, \mathrm{OCH}), 2.99\left(1 \mathrm{H}\right.$, quint, $\left.J 7.2, \mathrm{CHCH}_{3}\right), 2.73\left(2 \mathrm{H}\right.$, br t, $\left.J 7.4, \mathrm{CH}_{2} \mathrm{CO}_{2}\right), 2.61-$ $2.40\left(8 \mathrm{H}, \mathrm{m}, \mathrm{CH}_{2} \mathrm{~S}\right), 2.05-1.78\left(2 \mathrm{H}, \mathrm{m}, \mathrm{CH}_{2} \mathrm{CHOC}(\mathrm{O})\right), 1.29-1.12\left(9 \mathrm{H}, \mathrm{m}, \mathrm{SCH}_{2} \mathrm{CH}_{3} /\right.$ $\mathrm{CHCH}_{3}$ ); (Minor Diastereomer) 7.35-7.25 (2H, m, Ph), 7.21-7.12 (1H, m, Ph), 7.05-6.96 $(2 \mathrm{H}, \mathrm{m}, \mathrm{Ph}), 5.44-5.29(1 \mathrm{H}, \mathrm{m}, \mathrm{OCH}), 2.96-2.85\left(1 \mathrm{H}, \mathrm{m}, \mathrm{CHCH}_{3}\right), 2.73(2 \mathrm{H}$, br t, J 7.4, 
$\left.\mathrm{CH}_{2} \mathrm{CO}_{2}\right), 2.61-2.40\left(8 \mathrm{H}, \mathrm{m}, \mathrm{CH}_{2} \mathrm{~S}\right), 2.05-1.78\left(2 \mathrm{H}, \mathrm{m}, \mathrm{CH}_{2} \mathrm{CHOC}(\mathrm{O})\right), 1.29-1.12(9 \mathrm{H}, \mathrm{m}$, $\left.\mathrm{SCH}_{2} \mathrm{CH}_{3} / \mathrm{CHCH}_{3}\right) ; \delta_{\mathrm{C}}\left(75 \mathrm{MHz}, \mathrm{CDCl}_{3}\right)$ 172.4, 172.0, 171.9, 171.7, 150.92, 150.86, 130.0, 129.9, 126.4, 121.9, 121.8, 121.7, 115.7, 74.2, 73.7, 46.7, 43.4, 35.3, 32.5, 31.7, 28.0, 27.7, 26.9, 26.4, 26.3, 15.11, 15.06, 13.1, 12.2; $v_{\max }\left(\right.$ film) $/ \mathrm{cm}^{-1} 2968,2816,1758$, 1736, 1493; m/z, (CI+) $402\left(100 \%, \mathrm{M}+\mathrm{NH}_{4}\right), 385,291,268$; found $385.1504[\mathrm{M}+\mathrm{H}]^{+}$ $\mathrm{C}_{19} \mathrm{H}_{29} \mathrm{O}_{4} \mathrm{~S}_{2}$ requires 385.1502; (Found C, 59.4; H, 7.4. $\mathrm{C}_{19} \mathrm{H}_{28} \mathrm{O}_{4} \mathrm{~S}_{2}$ requires $\mathrm{C}, 59.3 ; \mathrm{H}$, $7.3)$.

\section{Preparation of 2-Methyl-5-methylsulfanyl-1-phenylpentane-1,3-dione (5)}

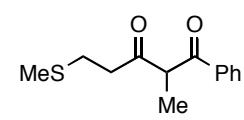

To a stirred solution of diisopropylamine $(0.78 \mathrm{~mL}, 582 \mathrm{mg}, 4.5 \mathrm{mmol})$ dissolved in THF (20 mL) cooled to $-78^{\circ} \mathrm{C}$ was added n-butyllithium (1.8 $\mathrm{mL}$ of a $2.5 \mathrm{M}$ hexanes solution) dropwise over 10 minutes. The reaction mixture was warmed to $0{ }^{\circ} \mathrm{C}$ for a then to the reaction mixture was added propiophenone $(0.6 \mathrm{~mL}, 4.5 \mathrm{mmol})$ over a 10 minute period. The reaction mixture was then warmed to room temperature a left for a further 30 minutes before a solution of methy-3-(methylthio)propionate $(0.56 \mathrm{~mL}, 604 \mathrm{mg}, 4.5$ mmol) dissolved in THF (10 mL) was added over 10 minutes. The reaction mixture was left overnight at room temperature before it was quenched with water $(10 \mathrm{~mL})$ and extracted with ether $(3 \times 30 \mathrm{~mL})$, the combined organic phases were dried $\left(\mathrm{MgSO}_{4}\right)$ and concentrated in vacuo to give a pale yellow oil which was purified further by column chromatography (9:1 hexane: EtOAc) to afford the title compound as a colorless oil (479 $\mathrm{mg}, 45 \%) ; \delta_{\mathrm{H}}\left(300 \mathrm{MHz}, \mathrm{CDCl}_{3}\right)$ 7.93-7.87 (2H, m, Ph), 7.57-7.50 (1H, m, Ph), $4.46(1 \mathrm{H}$, q, J 7.2, $\mathrm{C}(\mathrm{O}) \mathrm{CHC}(\mathrm{O})), 2.82-2.56\left(4 \mathrm{H}, \mathrm{m}, \mathrm{CH}_{2} \mathrm{CH}_{2} \mathrm{C}(\mathrm{O})\right), 1.98\left(3 \mathrm{H}\right.$, br s, $\left.\mathrm{SCH}_{3}\right), 1.39$ $\left(3 \mathrm{H}, \mathrm{d}, \mathrm{J} 7.2, \mathrm{CHCH}_{3}\right) ; \delta_{\mathrm{C}}\left(75 \mathrm{MHz}, \mathrm{CDCl}_{3}\right)$ 204.4, 196.3, 134.8, 132.8, 127.9, 127.7, $55.2,39.5,26.8,14.7,12.6 ; v_{\max }($ film $) / \mathrm{cm}^{-1} 2918,1719,1675,1448$. 
General procedure for three-component reductive aldol reactions: Preparation of tert-Butyl 3-cyano-2-(3-methylsulfanylpropionyloxy)butanoate (8)

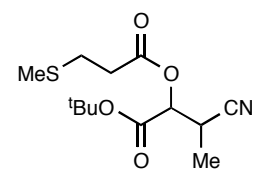

Hydrogen gas was bubbled through a stirred solution of $[\mathrm{Rh}(\mathrm{nbd}) \mathrm{dppe}] \mathrm{ClO}_{4}(10 \mathrm{mg}$, $0.014 \mathrm{mmol})$ dissolved in DCE $(10 \mathrm{~mL})$ for 2 minutes, during this time a color change from dark orange to yellow was realised, the solution was then purged with argon for a further 0.5 minutes. To this mixture was next added 3-(methylthio)propionaldehyde (14 $\mu \mathrm{L}, 15 \mathrm{mg}, 0.14 \mathrm{mmol}$ ) follwed by tert-butyl glyoxylate ${ }^{3}(18 \mathrm{mg}, 0.14 \mathrm{mmol})$ and acrylonitrile $(18 \mu \mathrm{L}, 15 \mathrm{mg}, 0.28 \mathrm{mmol})$. The reaction mixture was then heated to $70{ }^{\circ} \mathrm{C}$ for 16 hours. The crude reaction mixture was concentrated in vacuo and purified by column chromatography (9:1 Hexanxe: EtOAc) to give the product as a colorless oil (25 $\mathrm{mg}, 60 \%)$ as a mixture of diastereomers $(1.4: 1) ; \delta_{\mathrm{H}}\left(300 \mathrm{MHz}, \mathrm{CDCl}_{3}\right)(\mathrm{Major}$ Diastereomer) 5.07 (1H, d, J 4.1, OCH), 3.27-3.13 (1H, m, CHCN), 2.80-2.67 (4H, m, $\left.\mathrm{CH}_{2} \mathrm{CH}_{2} \mathrm{~S}\right), 2.08$ (3H, br s, $\left.\mathrm{SCH}_{3}\right), 1.43(9 \mathrm{H}, \mathrm{s}, t-\mathrm{Bu}), 1.34\left(3 \mathrm{H}, \mathrm{d}, J\right.$ 7.2, $\left.\mathrm{CH}_{3} \mathrm{CH}\right)$; (Minor Diastereomer) 4.95 (1H, d, J 4.1, OCH), 3.27-3.13 (1H, m, CHCN), 2.80-2.67 (4H, m, $\left.\mathrm{CH}_{2} \mathrm{CH}_{2} \mathrm{~S}\right), 2.08,2.08\left(3 \mathrm{H}\right.$, br s, $\left.\mathrm{SCH}_{3}\right), 1.44(9 \mathrm{H}, \mathrm{s}, t-\mathrm{Bu}), 1.34\left(3 \mathrm{H}, \mathrm{d}, J 7.5, \mathrm{CH}_{3} \mathrm{CH}\right)$; $\delta_{\mathrm{C}}\left(75 \mathrm{MHz}, \mathrm{CDCl}_{3}\right)$ 171.2, 171.1, 166.0, 165.7, 119.5, 119.0, 84.6, 72.5, 72.0, 34.44, $34.39,29.14,29.09,28.5,28.3,28.2,15.92,15.87,15.0,13.7 ; v_{\max }($ film $) / \mathrm{cm}^{-1} 2965$, 2922, 1747, 1368; $\mathrm{m} / \mathrm{z}$ (CI+) 305 (100\%, M+NH$), 288,249,187$; found 305.1528 $\left[\mathrm{M}+\mathrm{NH}_{4}\right]^{+} \mathrm{C}_{13} \mathrm{H}_{25} \mathrm{O}_{4} \mathrm{~N}_{2} \mathrm{~S}$ requires 305.1530.

\section{Preparation of tert-Butyl 3-methyl-2-(3-methylsulfanylpropionyloxy)-4-oxo-4- phenylbutanoate (9)}

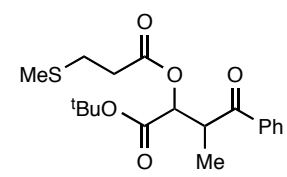

The general procedure for three component coupling was employed substituting phenyl vinyl ketone ( $36 \mu \mathrm{L}, 37 \mathrm{mg}, 0.28 \mathrm{mmol})$ for acrylonitrile to give the product as a 
colorless oil (51 mg, $99 \%)$ as a mixture of diastereomers $(2.1: 1) ; \delta_{\mathrm{H}}\left(300 \mathrm{MHz}, \mathrm{CDCl}_{3}\right)$ (Major Diastereomer) 7.93-7.85 (2H, m, Ph), 7.58-7.48 (1H, m, Ph), 7.47-7.38 (2H, m, $\mathrm{Ph}), 5.35$ (1H, d, J 5.3, OCH), 3.98 (1H, dt, J 7.2, 6.8, $\left.\mathrm{CH}_{2} \mathrm{CHCHO}\right), 2.74-2.45$ (4H, m, $\left.\mathrm{CH}_{2} \mathrm{CH}_{2} \mathrm{~S}\right), 2.04\left(3 \mathrm{H}, \mathrm{s}, \mathrm{SCH}_{3}\right), 1.34(9 \mathrm{H}, \mathrm{s}, t-\mathrm{Bu}), 1.21\left(3 \mathrm{H}, \mathrm{d}, J 6.8, \mathrm{CH}_{3} \mathrm{CH}\right)$; (Minor Diastereomer) 7.93-7.85 (2H, m, Ph), 7.58-7.48 (1H, m, Ph), 7.47-7.38 (2H, m, Ph), 5.16 $(1 \mathrm{H}, \mathrm{d}, J 5.3, \mathrm{OCH}), 3.88\left(1 \mathrm{H}, \mathrm{dt}, J 7.2,6.8, \mathrm{CH}_{2} \mathrm{CHCHO}\right), 2.74-2.45\left(4 \mathrm{H}, \mathrm{m}, \mathrm{CH}_{2} \mathrm{CH}_{2} \mathrm{~S}\right)$, $1.99(3 \mathrm{H}, \mathrm{s}, \mathrm{SCH}), 1.38(9 \mathrm{H}, \mathrm{s}, t-\mathrm{Bu}), 1.19\left(3 \mathrm{H}, \mathrm{d}, J 6.8, \mathrm{CH}_{3} \mathrm{CH}\right) ; \delta_{\mathrm{C}}\left(75 \mathrm{MHz}, \mathrm{CDCl}_{3}\right)$ $198.4,170.0,169.9,166.9,166.7,135.1,134.7,132.3,132.3,127.8,127.5,127.42$, $127.37,127.25,81.90,81.88,73.36,73.32,42.9,41.2,41.0,33.3,33.1,28.1,27.8,27.7$, 26.9, 26.8, 14.43, 14.37, 11.9, 11.5, 10.8; $v_{\max }$ (film) / $\mathrm{cm}^{-1} 2979,2920,1743,1687$, $1156 ; \mathrm{m} / \mathrm{z}(\mathrm{CI}+) 384\left(100 \%, \mathrm{M}+\mathrm{NH}_{4}\right), 328,264,208$; found $367.1576[\mathrm{M}+\mathrm{H}]^{+} \mathrm{C}_{19} \mathrm{H}_{27} \mathrm{O}_{5} \mathrm{~S}$ requires 367.1574; (Found $\mathrm{C}, 61.6 ; \mathrm{H}, 7.2 . \mathrm{C}_{19} \mathrm{H}_{26} \mathrm{O}_{5} \mathrm{~S}$ requires $\mathrm{C}, 62.3 ; \mathrm{H}, 7.15$ ).

\section{Preparation of $\left(\mathrm{C}_{1}\left\{{ }^{2} \mathrm{H}\right\}\right)$-3-(methylthio)propionaldehyde}

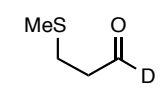

To a stirred suspension of $\mathrm{LiAlD}_{4}(1.56 \mathrm{~g}, 37.3 \mathrm{mmol})$ in ether $(100 \mathrm{~mL})$ cooled to -20 ${ }^{\circ} \mathrm{C}$ was added methy-3-(methylthio)propionate $(4.7 \mathrm{~mL}, 5.00 \mathrm{~g}, 37.3 \mathrm{mmol}$ ) over a 30 minute period. The reaction mixture was warmed to room temperature over a 2 hour period. The reaction was then quenched carefully with $\mathrm{D}_{2} \mathrm{O}(4 \mathrm{~mL})$ and the resulting suspension was dried over $\mathrm{MgSO}_{4}$, the resultant slurry was filtered and washed with ether $(2 \times 25 \mathrm{~mL})$. The combined organic washings were concentrated in vacuo to afford $\left(\mathrm{C}_{1}\left\{{ }^{2} \mathrm{H}_{2}\right\}, \mathrm{O}\left\{{ }^{2} \mathrm{H}\right\}\right)-3-($ methylthio)propanol as a colourless oil. This material was used immediatedly without further purification. To a solution of the $\left(\mathrm{D}_{3}\right)-3$ (methylthio)propanol $(0.5 \mathrm{~g}, 4.5 \mathrm{mmol})$ dissolved in DCM $(10 \mathrm{~mL})$ at room temperature was added Dess-Martin periodinane (2.04 g, $4.81 \mathrm{mmol})$ over a 1 hour period. Careful monitoring of the reaction by TLC ensured no over oxidation. Once the reaction was complete the crude reaction mixture was purified by column chromatography $(100 \%$ DCM) to afford the title compound as a colorless oil (330 mg, $67 \%$ ). This material was used immediately in further reactions; $\delta_{\mathrm{H}}\left(300 \mathrm{MHz}, \mathrm{CDCl}_{3}\right) 2.77-2.65(4 \mathrm{H}, \mathrm{m}$, 
$\left.\mathrm{CH}_{2} \mathrm{CH}_{2} \mathrm{~S}\right), 2.07\left(3 \mathrm{H}, \mathrm{s}, \mathrm{SCH}_{3}\right) ; \delta_{\mathrm{C}}\left(75 \mathrm{MHz} \mathrm{CDCl}_{3}\right) 200.7(\mathrm{t}, J 27.2, \mathrm{C}(\mathrm{O}) \mathrm{D}), 43.6(\mathrm{t}, J$ 3.8, $\left.\mathrm{CH}_{2} \mathrm{C}(\mathrm{O})\right), 26.8,16.0 ; v_{\max }\left(\right.$ film) $/ \mathrm{cm}^{-1} 2099,1641$.

\section{Preparation of $\left(\mathrm{C}_{5}\left\{{ }^{2} \mathrm{H}\right\}\right)$-tert-Butyl 3-methyl-2-(3-methylsulfanylpropionyloxy)-4-oxo-}

\section{4-phenylbutanoate (11)}

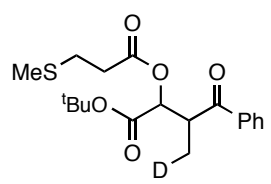

The general procedure for the three-component coupling was employed utilising the $\left(\mathrm{C}_{1}\left\{{ }^{2} \mathrm{H}\right\}\right)$-aldehyde and substituting phenyl vinyl ketone $(36 \mu \mathrm{L}, 37 \mathrm{mg}, 0.28 \mathrm{mmol})$ for acrylonitrile, to give the product as a colorless oil (52 $\mathrm{mg}, 99 \%)$ as a mixture of diastereomers (2.1:1); $\delta_{\mathrm{H}}\left(300 \mathrm{MHz}, \mathrm{CDCl}_{3}\right)$ (Major Diastereomer) 7.93-7.85 (2H, m, Ph), 7.58-7.48 (1H, m, Ph), 7.47-7.38 (2H, m, Ph), 5.35 (1H, d, J 5.3, OCH), 3.98 (1H, dt, J 7.2, 6.8, CH2CHCHO), 2.74-2.45 (4H, m, $\left.\mathrm{CH}_{2} \mathrm{CH}_{2} \mathrm{~S}\right), 2.04\left(3 \mathrm{H}, \mathrm{s}, \mathrm{SCH}_{3}\right), 1.34$ (9H, s, $t$ $\mathrm{Bu}), 1.21$ (2H, m, $\left.\mathrm{CH}_{2}{ }^{2} \mathrm{HCH}\right)$; (Minor Diastereomer) 7.93-7.85 (2H, m, Ph), 7.58-7.48 (1H, m, Ph), 7.47-7.38 (2H, m, Ph), 5.16 (1H, d, J 5.3, OCH), 3.88 (1H, dt, J 7.2, 6.8, $\left.\mathrm{CH}_{2} \mathrm{CHCHO}\right), 2.74-2.45$ (4H, m, $\left.\mathrm{CH}_{2} \mathrm{CH}_{2} \mathrm{~S}\right), 1.99\left(3 \mathrm{H}, \mathrm{s}, \mathrm{SCH}_{3}\right), 1.38$ (9H, s, $t$-Bu), 1.19 $\left(2 \mathrm{H}, \mathrm{m}, \mathrm{CH}_{2}{ }^{2} \mathrm{HCH}\right) ; \delta_{\mathrm{C}}\left(75 \mathrm{MHz}, \mathrm{CDCl}_{3}\right)$ 198.4, 170.0, 169.94, 169.88, 166.7, 135.1, $134.7,132.35,132.32,127.8,127.5,127.42,127.37,81.9,73.4,72.3,44.2,41.1,40.9$, $33.2,33.1,27.8,27.7,26.9,26.8,14.43,14.38,11.9,11.6,11.3\left(\mathrm{t}, J 19.6, \mathrm{CH}_{2} \mathrm{DCH}\right) ; v_{\max }$ (film) / $\mathrm{cm}^{-1} 2979,2920,1742$, 1688, 1154; (Found C, 61.8; H, 7.1. $\mathrm{C}_{19} \mathrm{H}_{25} \mathrm{DO}_{5} \mathrm{~S}$ requires C, 62.1; H, 7.4).

\section{References}

1. Weber H., Chemische Werke Huels GmbH, DE855704.

2. Schrock, R. R.; Osborn, J. A. J. Am. Chem. Soc. 1971, 93, 2397.

3. Bishop, J. E.; O’Connel, J. F.; Rapoport, H. J. Org. Chem. 1991, 56, 5079. 


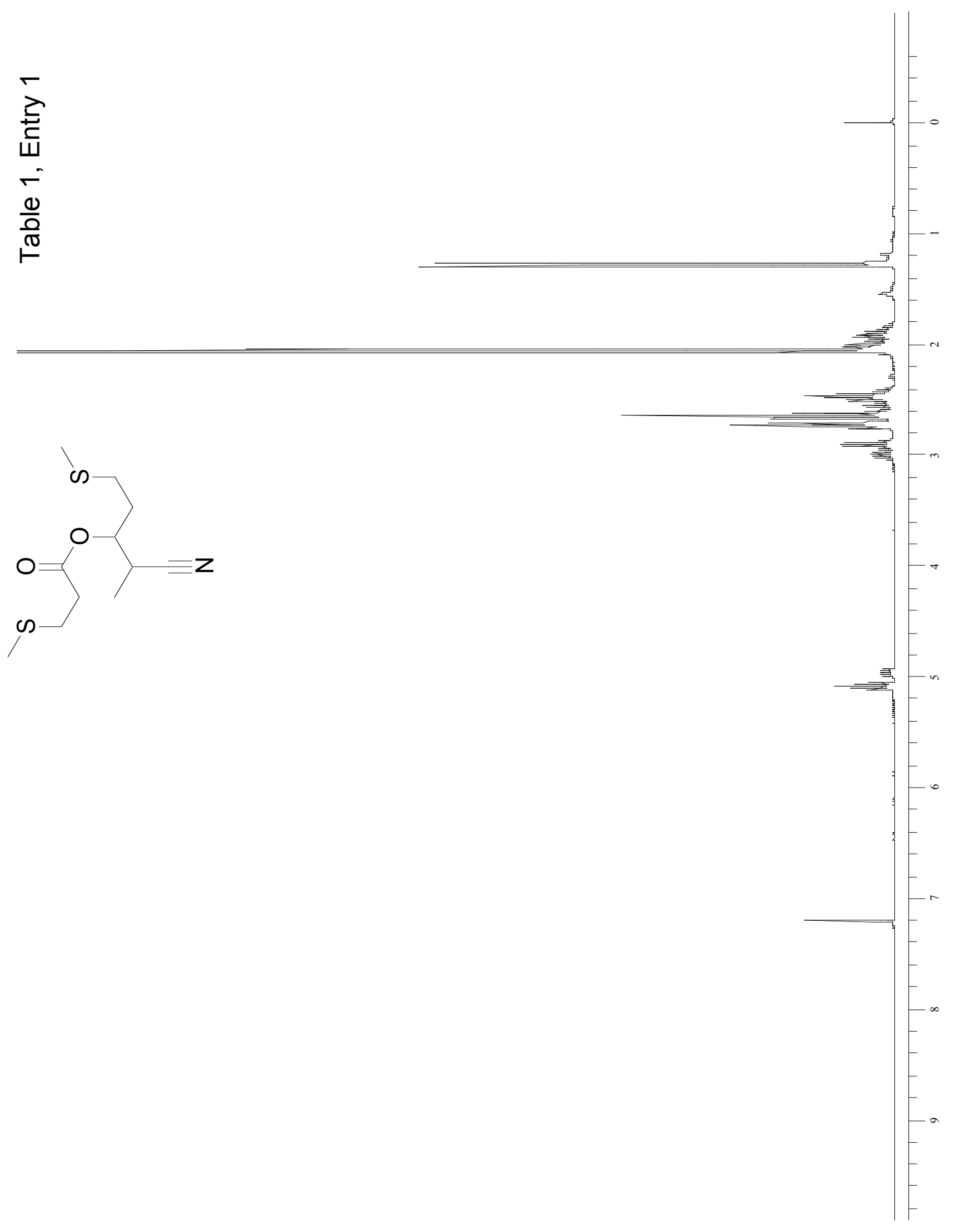




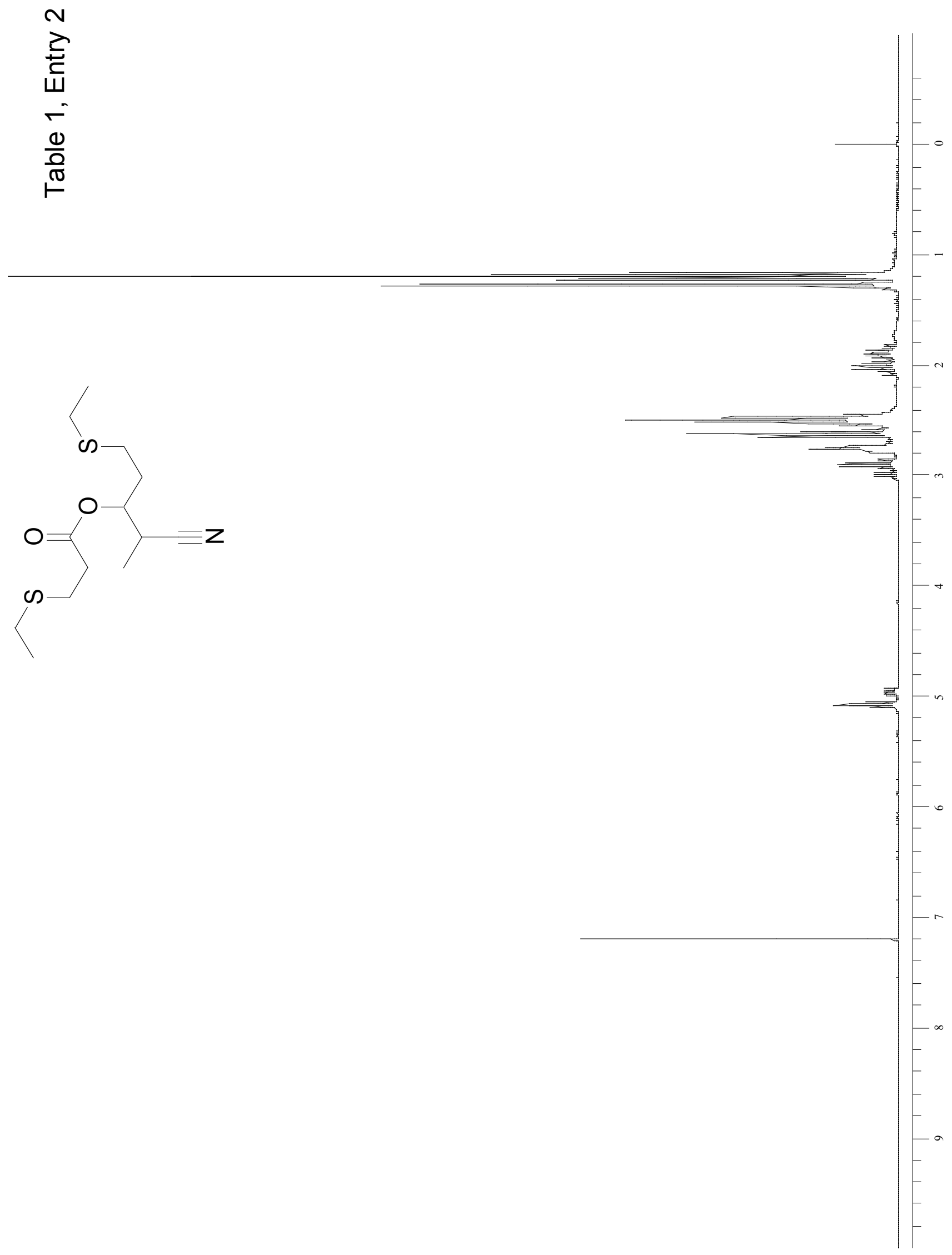




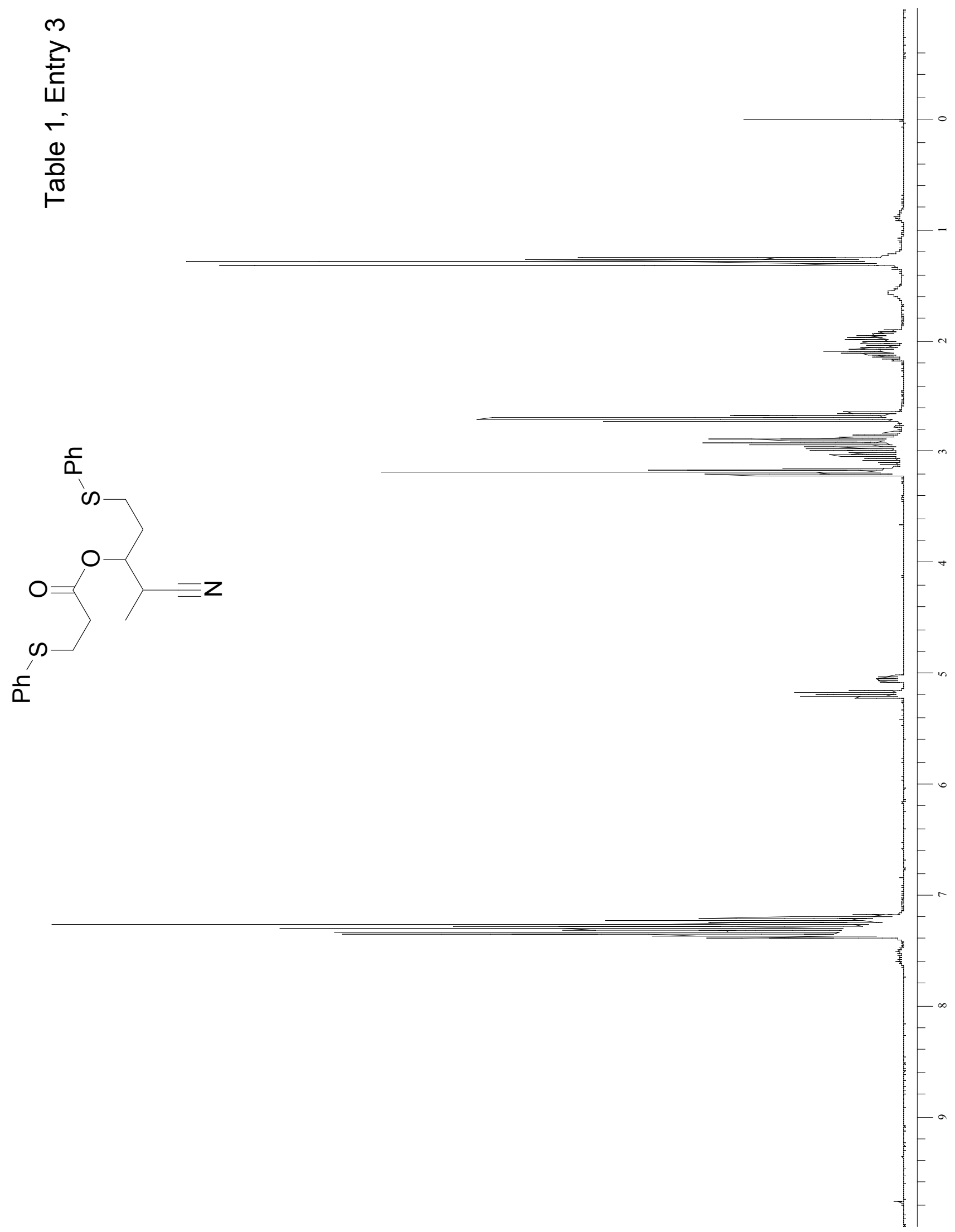



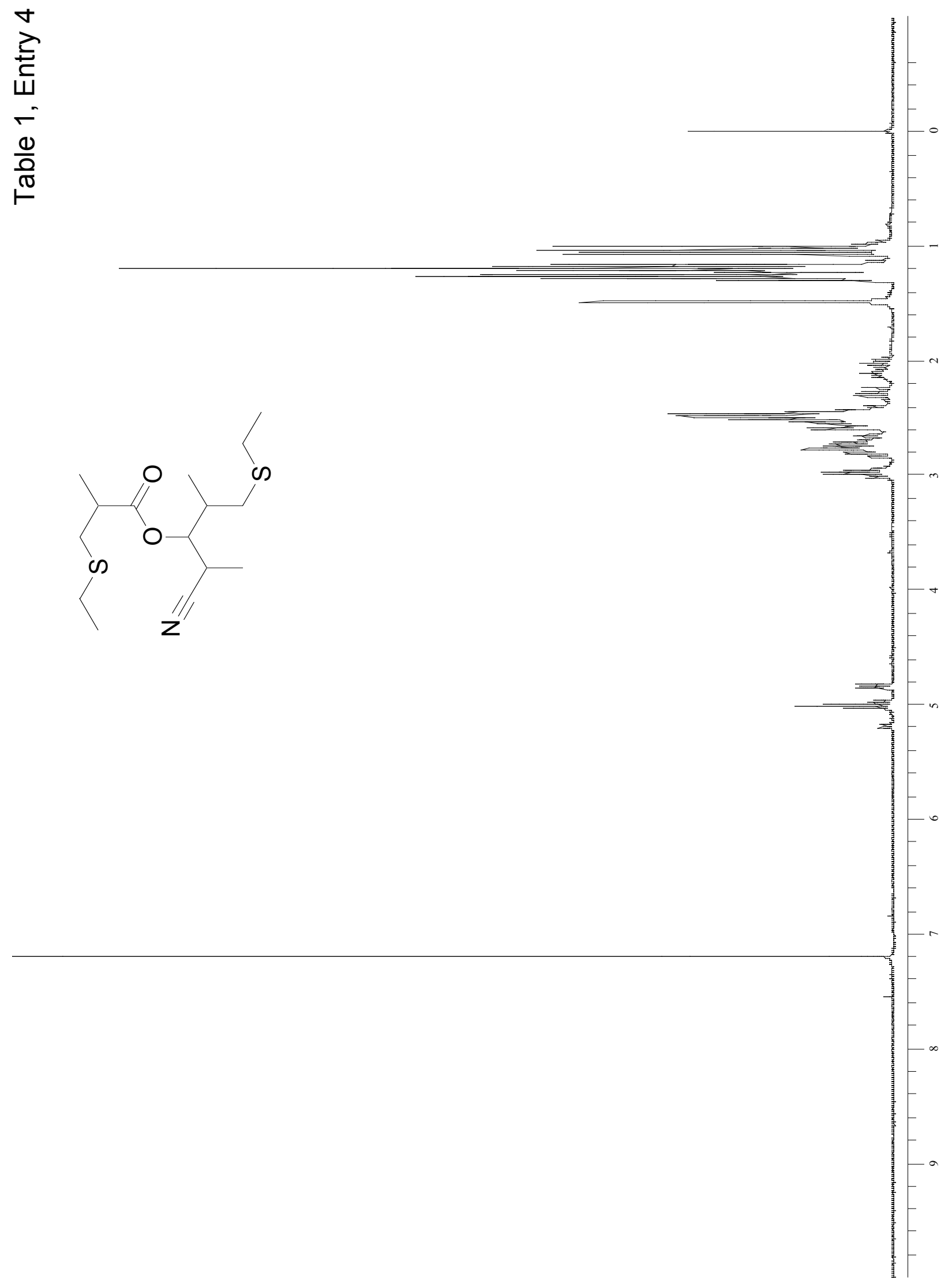


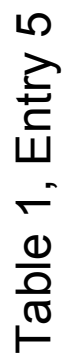
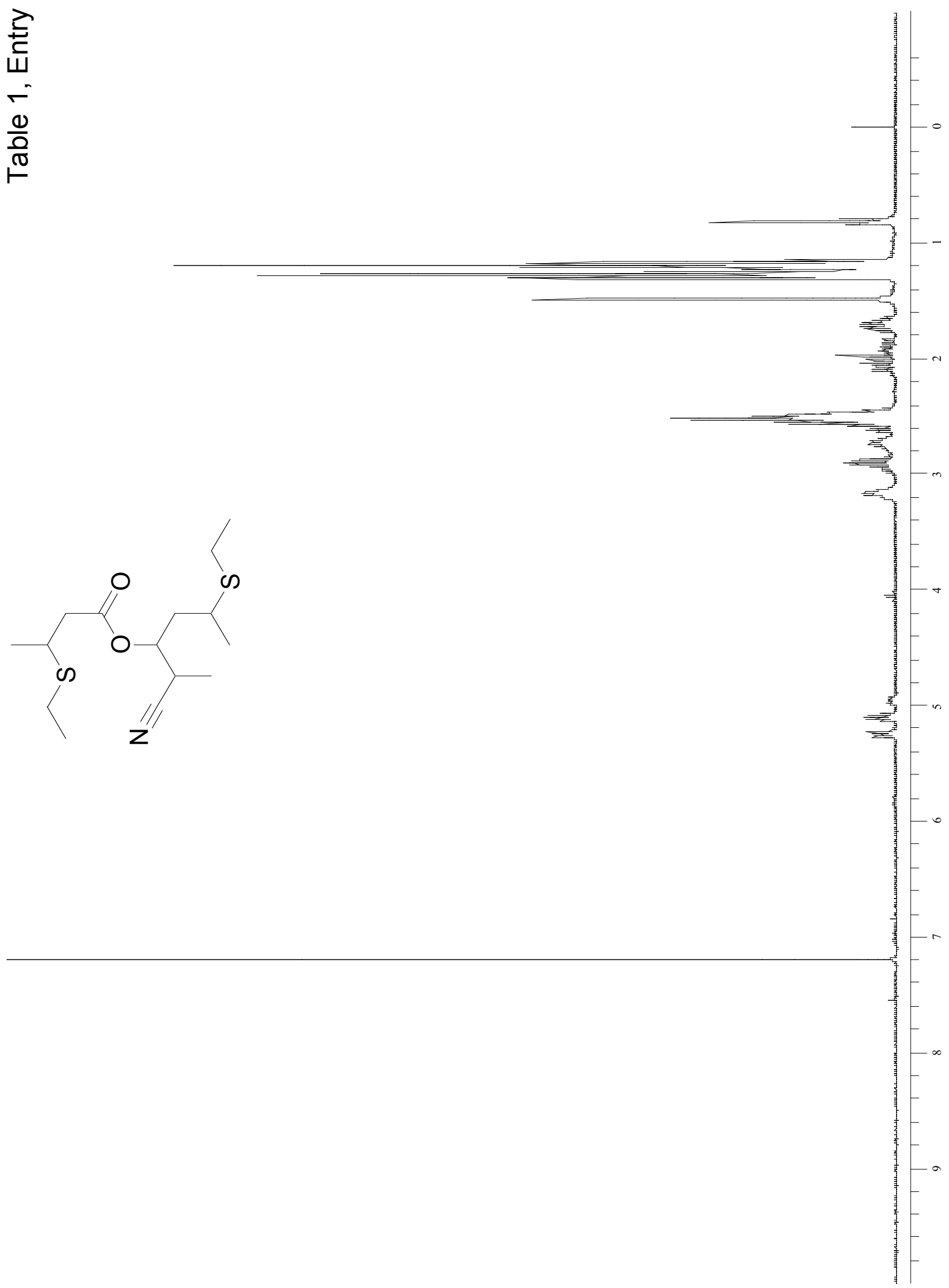

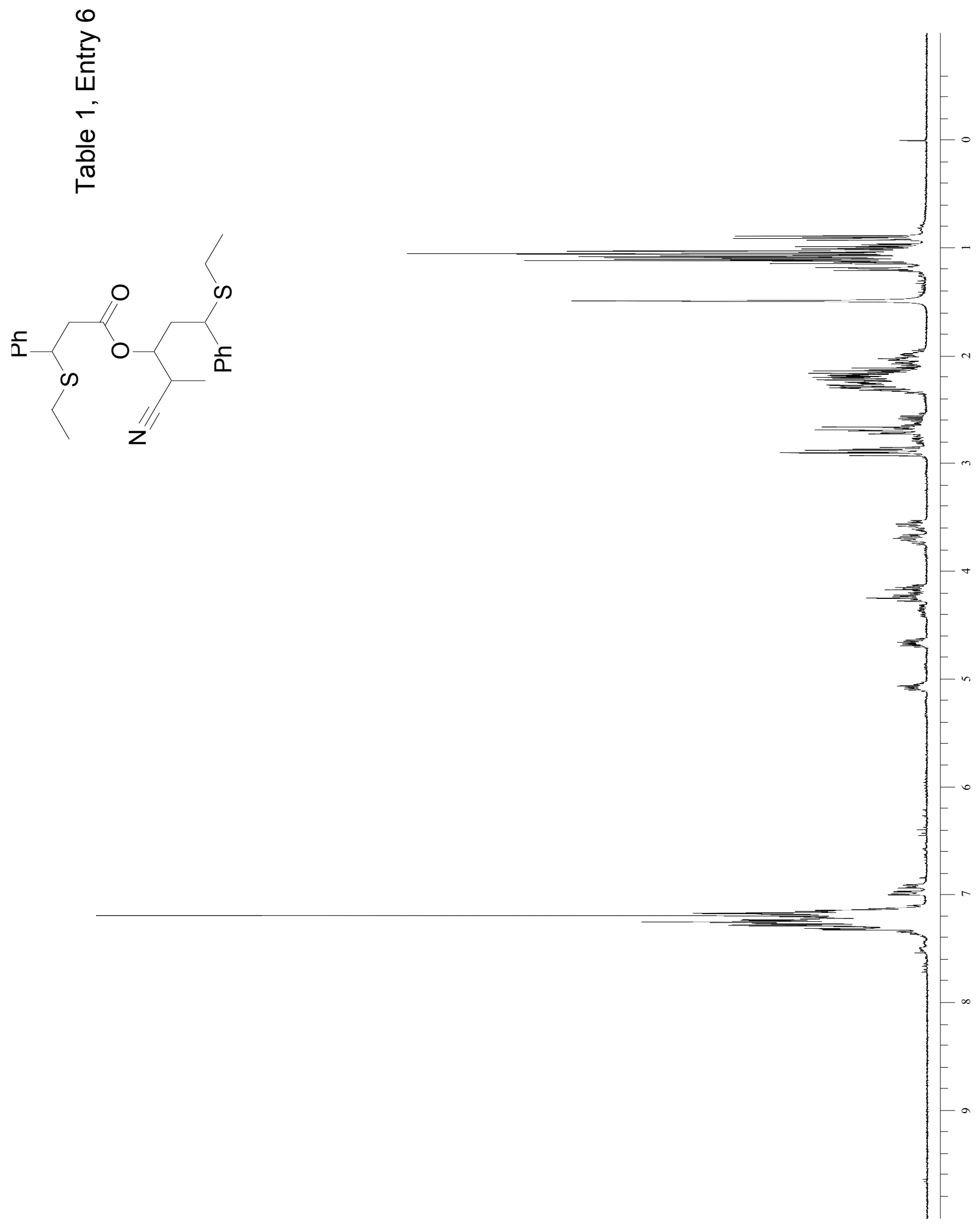


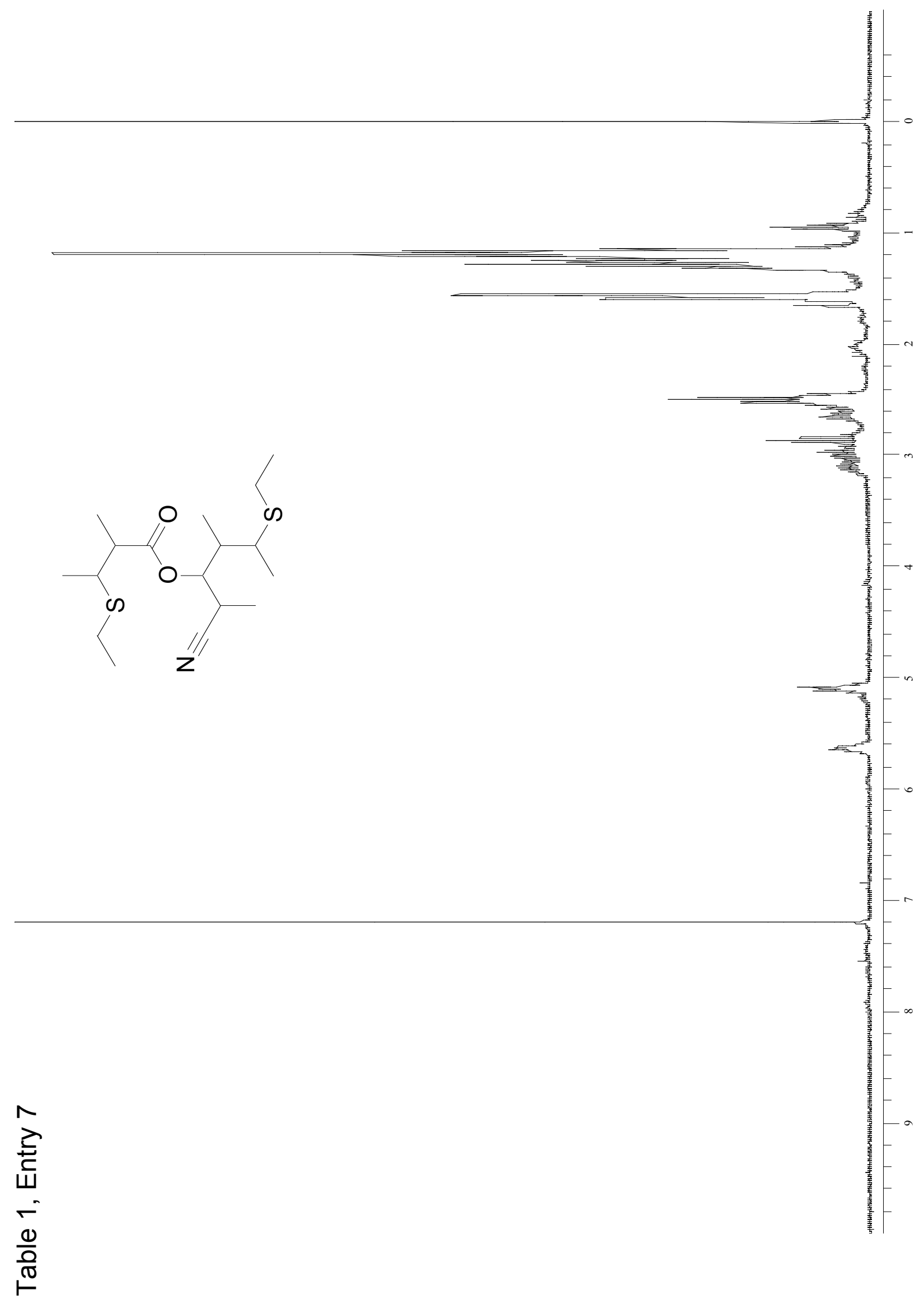




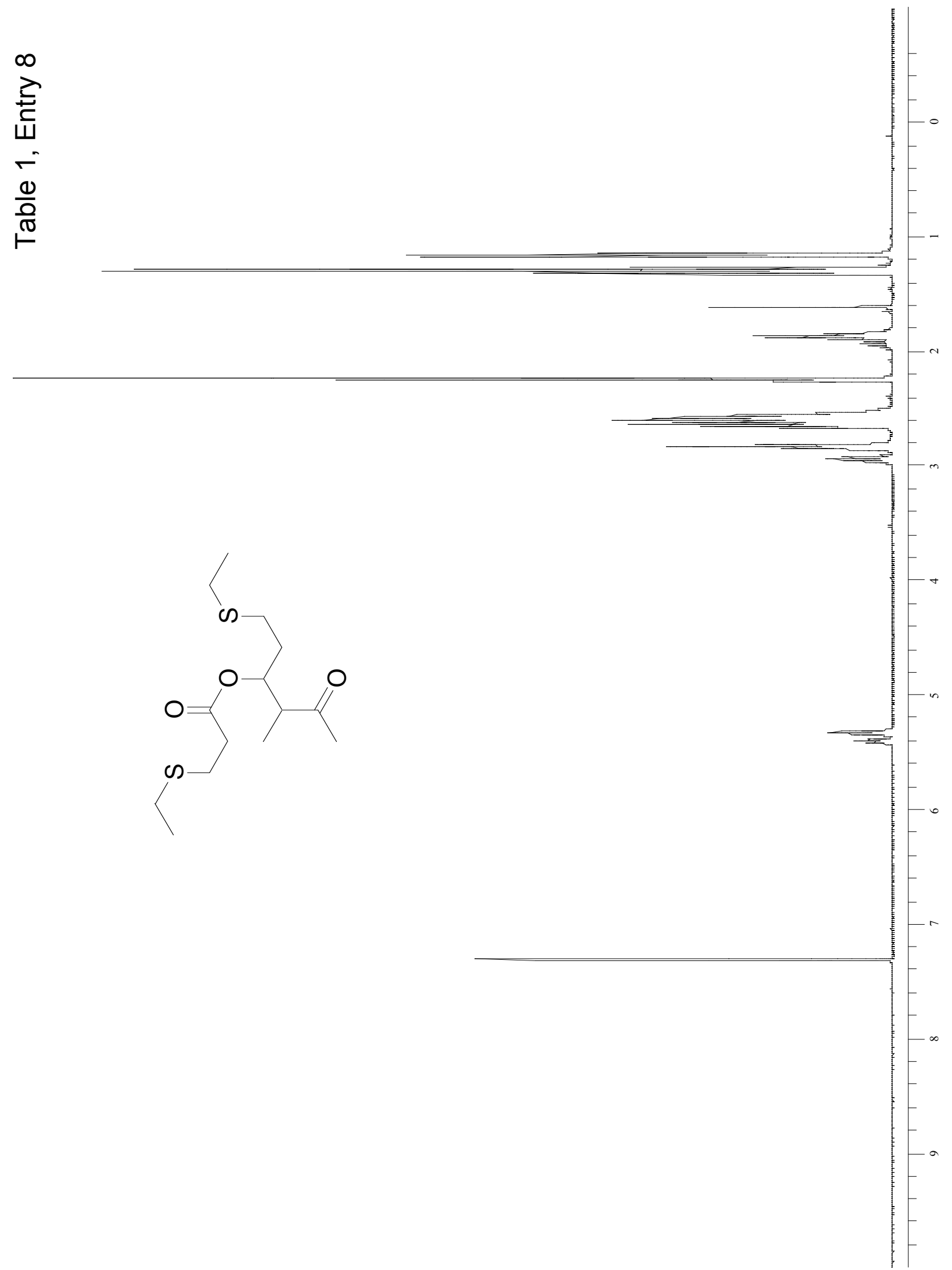




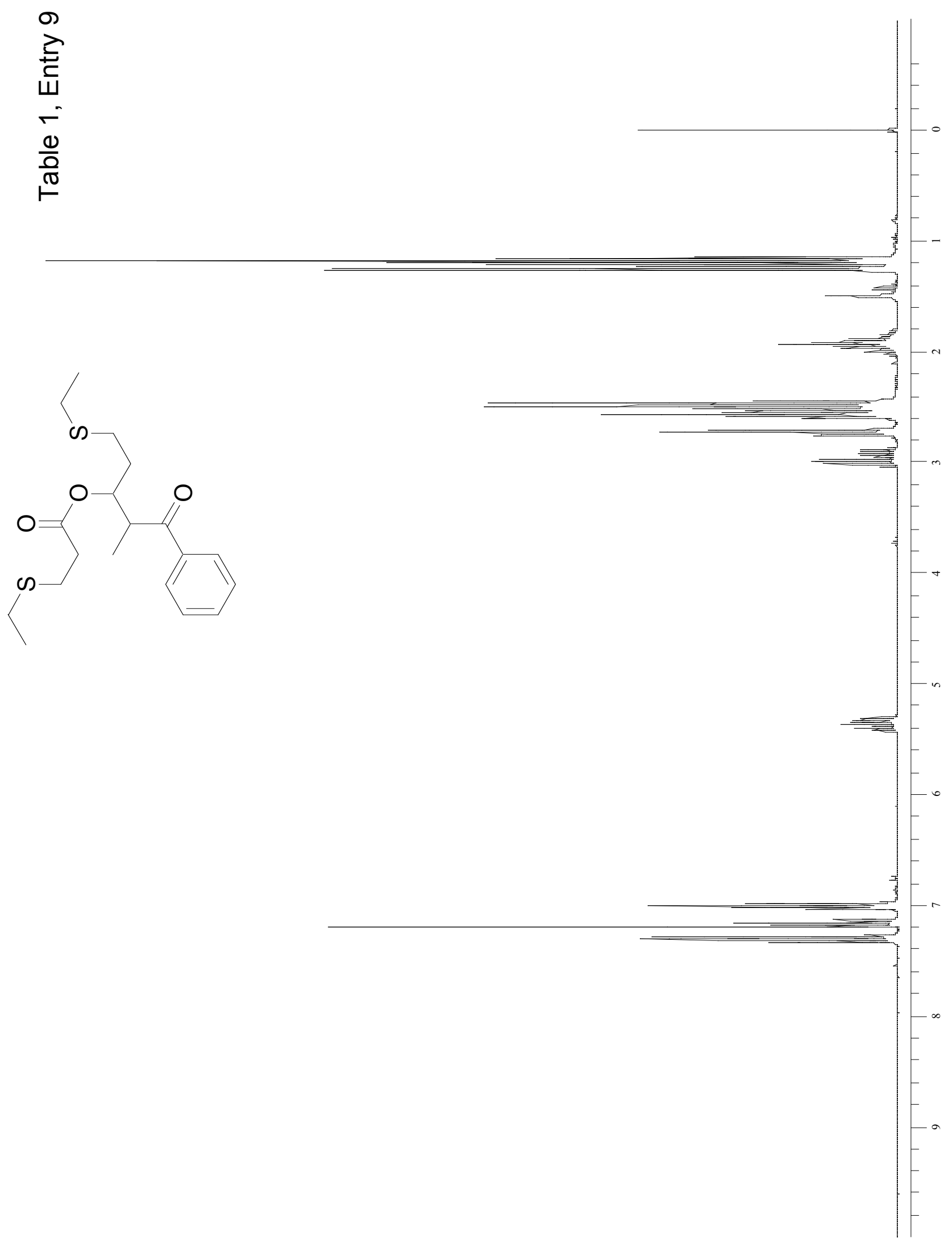




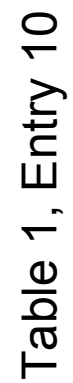
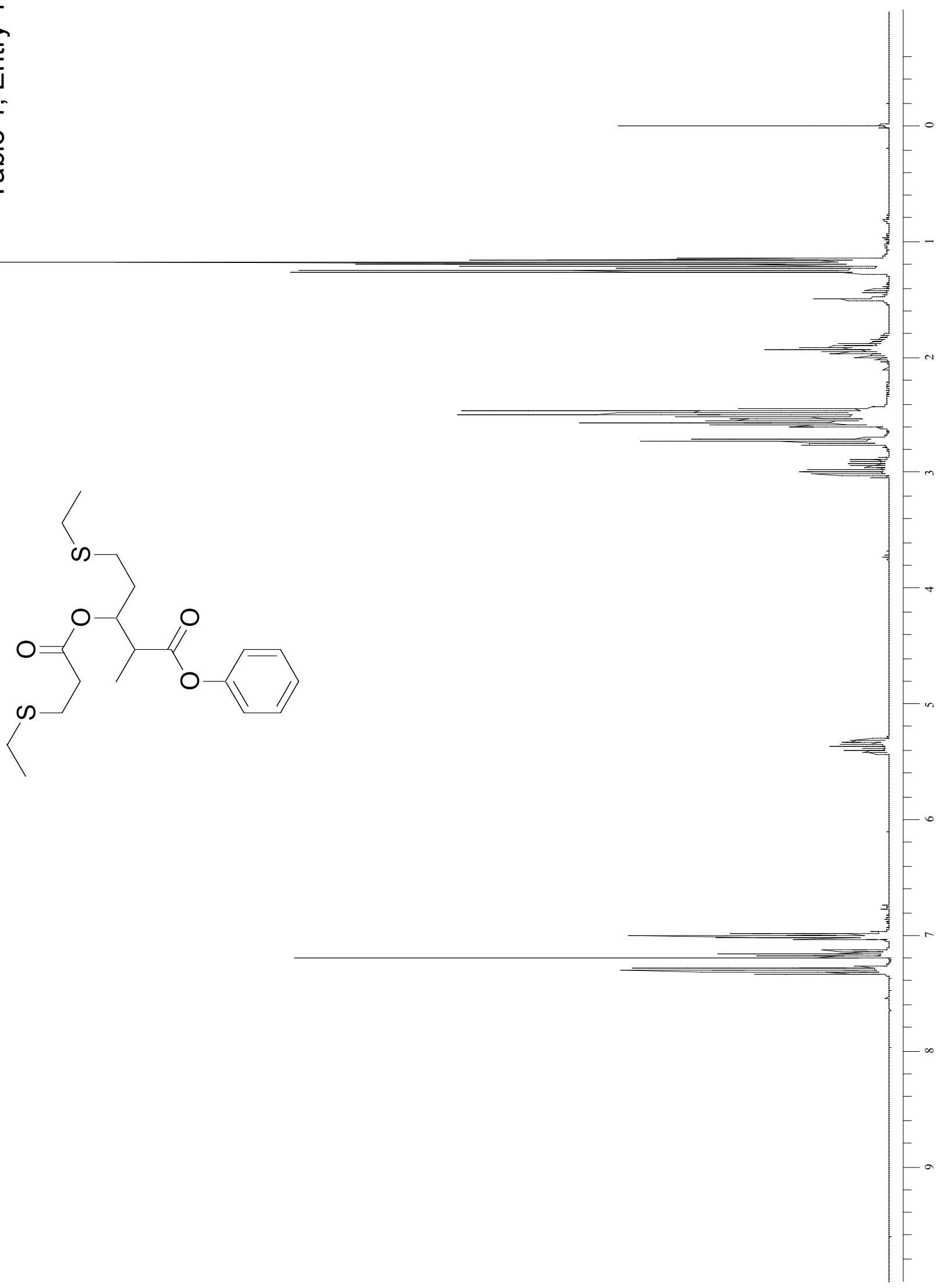


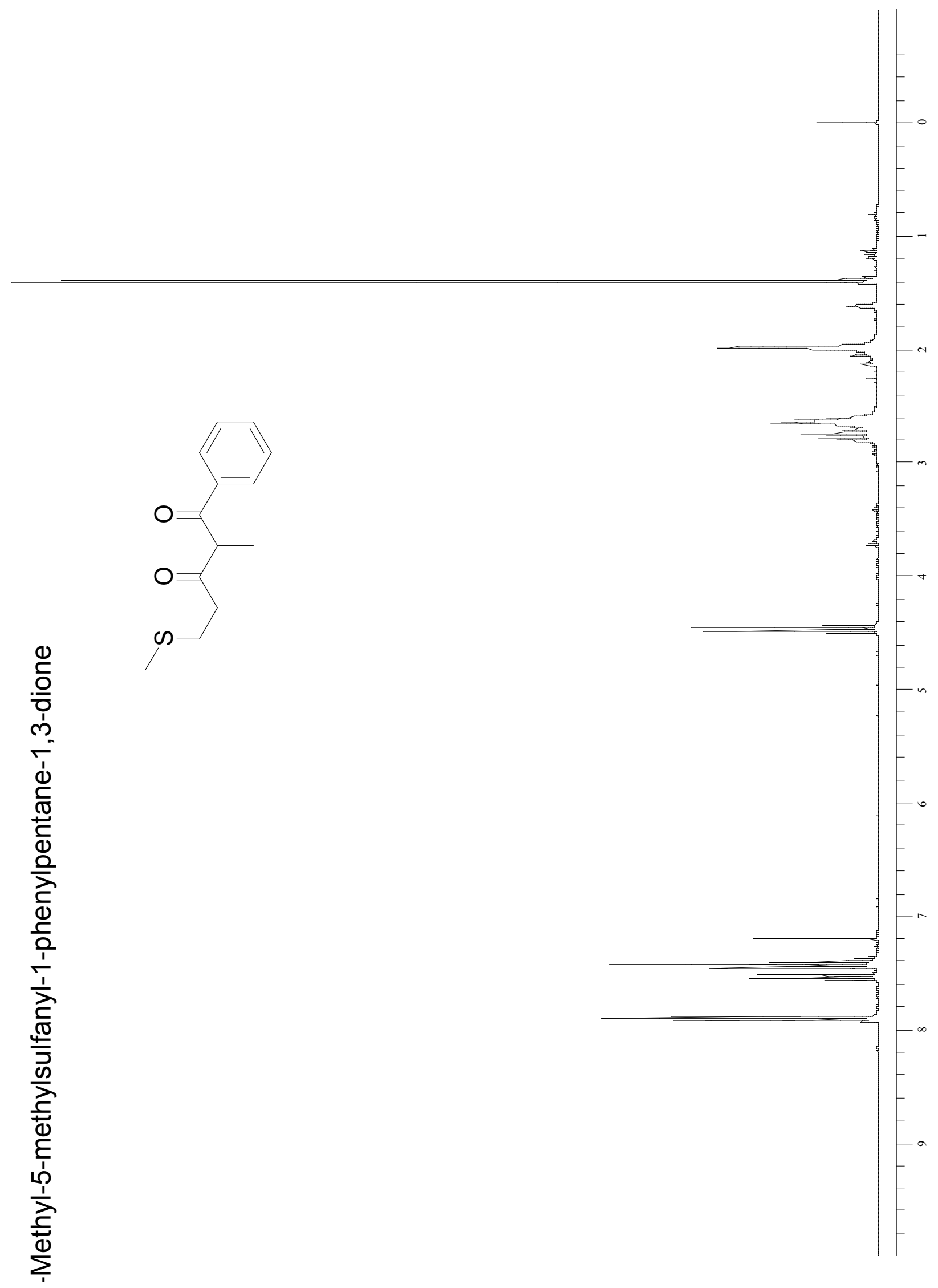


J. Am. Chem. Soc. Supporting Information

$S 23$

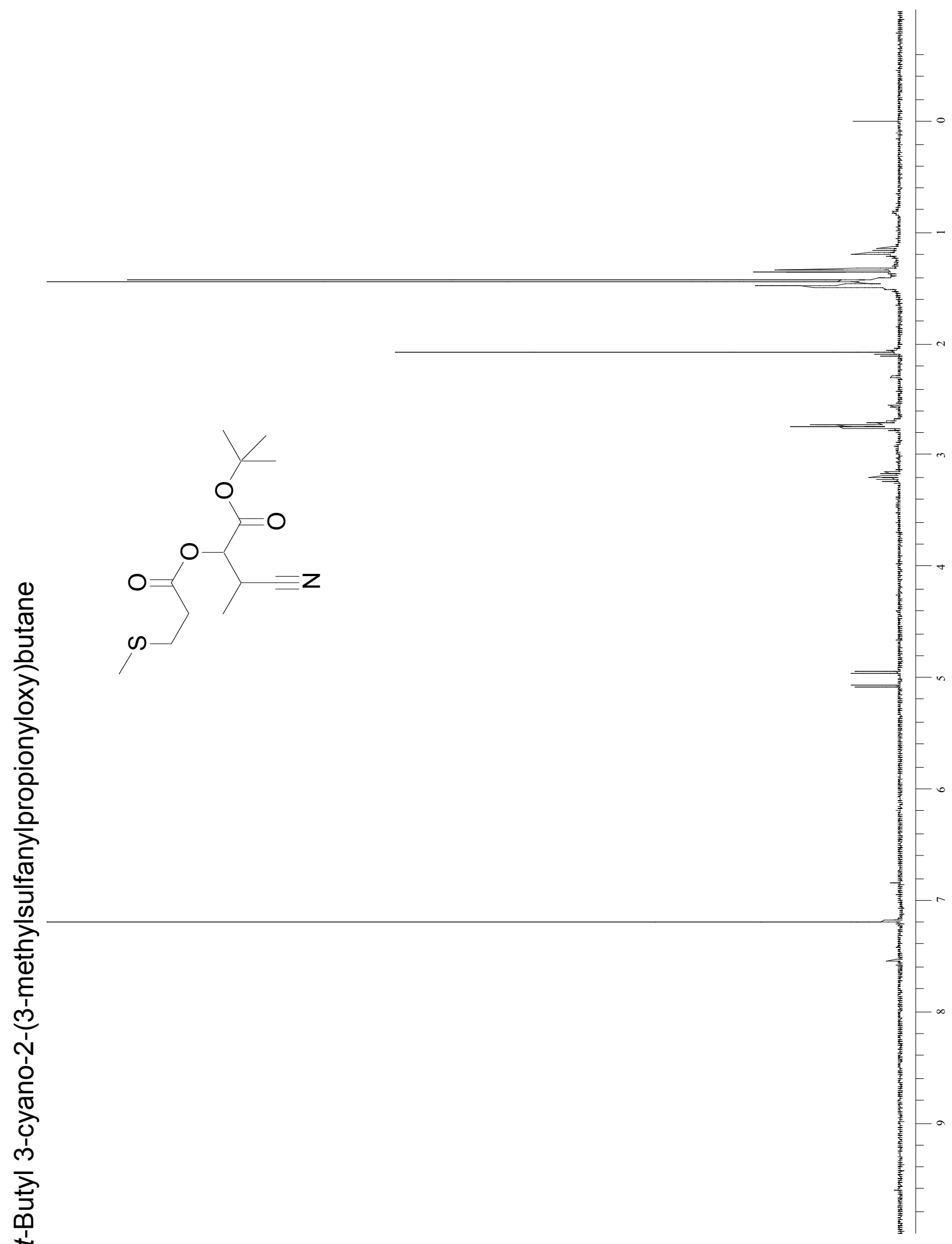




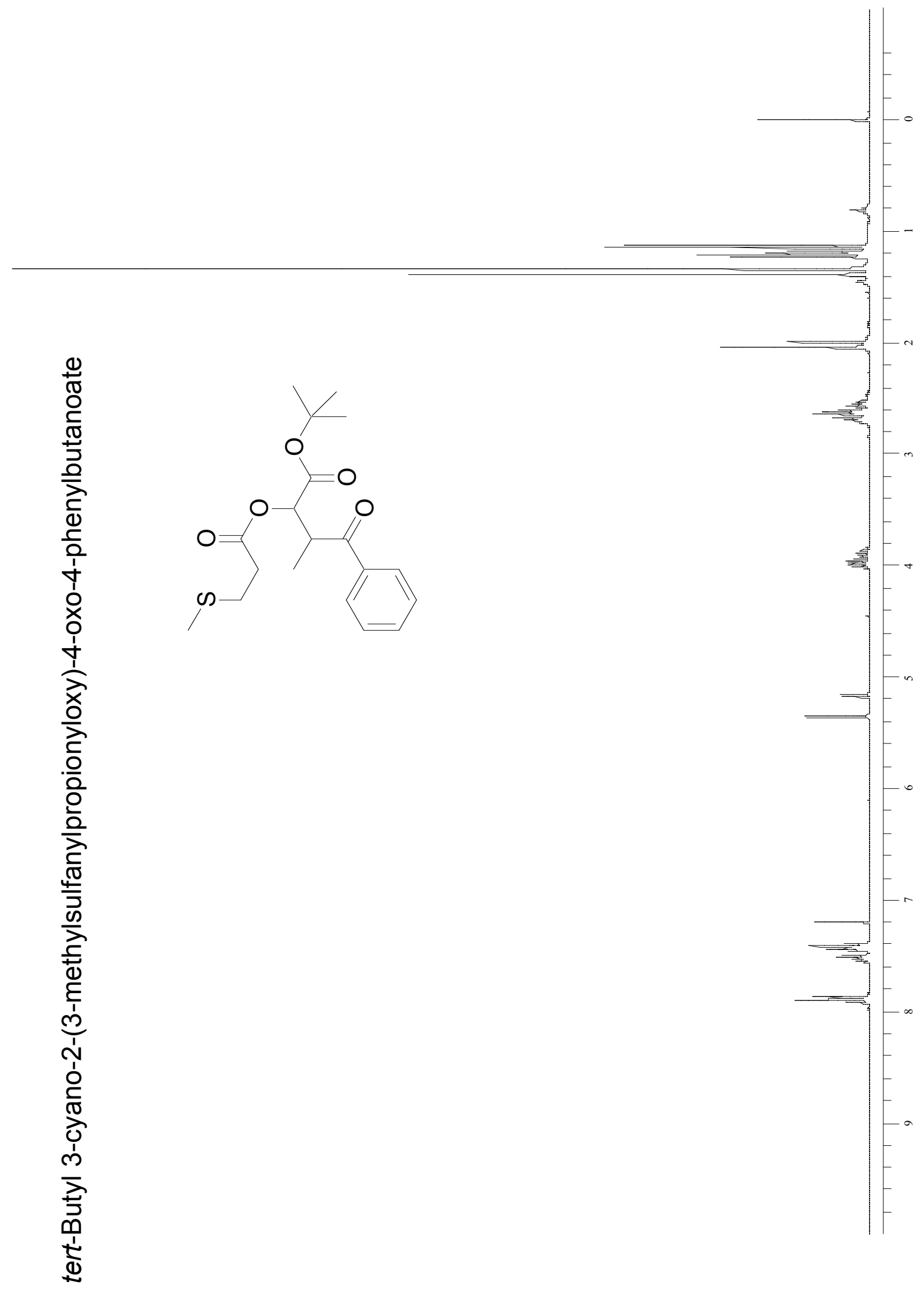




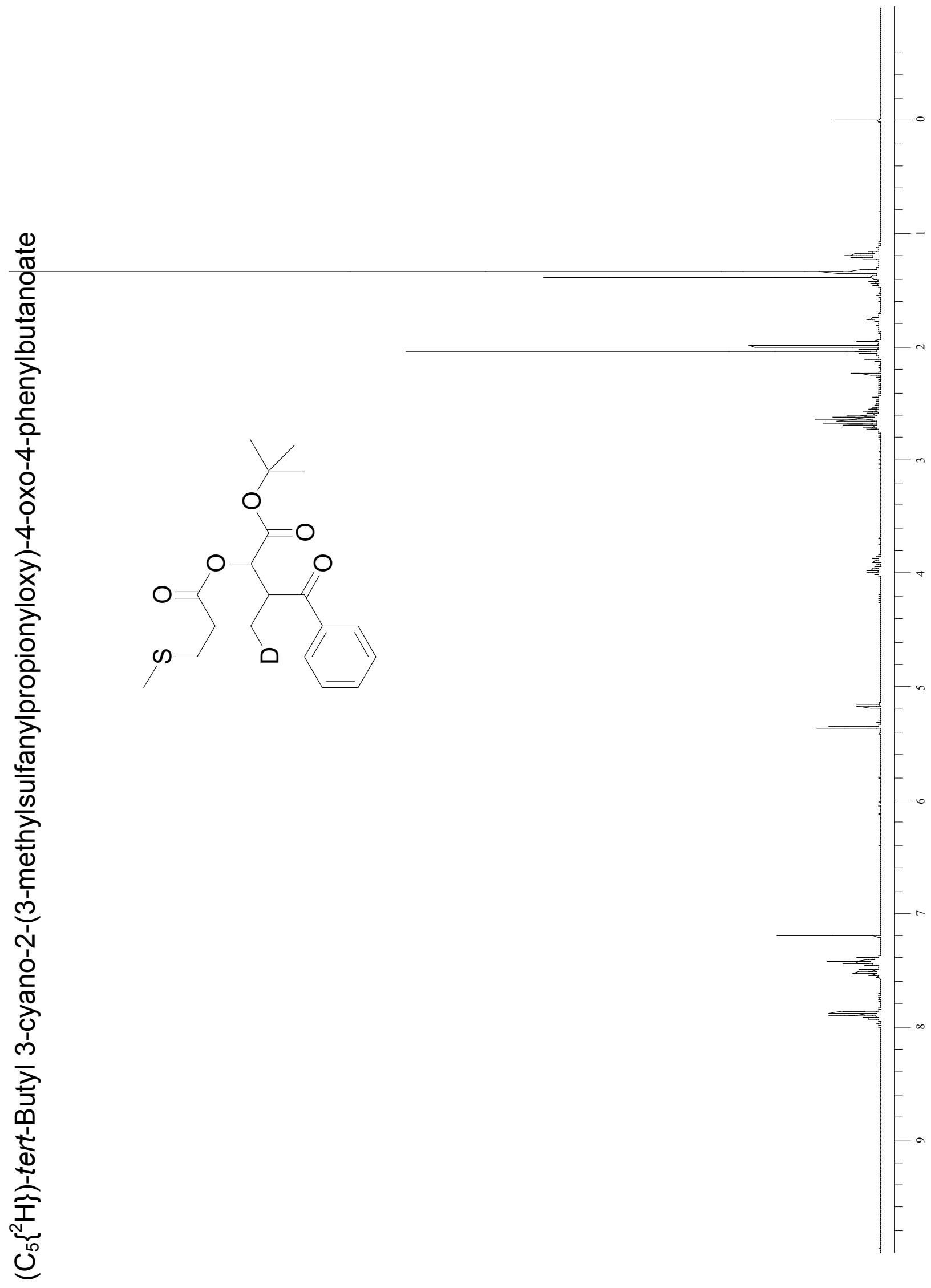

